\title{
Separable Joint Blind Deconvolution and Demixing
}

\author{
Dana Weitzner and Raja Giryes
}

\begin{abstract}
Blind deconvolution and demixing is the problem of reconstructing convolved signals and kernels from the sum of their convolutions. This problem arises in many applications, such as blind MIMO. This work presents a separable approach to blind deconvolution and demixing via convex optimization. Unlike previous works, our formulation allows separation into smaller optimization problems, which significantly improves complexity. We develop recovery guarantees, which comply with those of the original non-separable problem, and demonstrate the method performance under several normalization constraints.
\end{abstract}

Index Terms-Blind deconvolution, demixing, low-rank.

\section{INTRODUCTION}

$\mathbf{C}$ ONSIDER the task of restoring signals from a mixture of their bilinear measurements, involving unknown environment parameters. This problem is referred to as blind deconvolution and demixing, where signals are convolved with unknown kernels. It appears in various domains, e.g., audio and image processing [1]-[3] and wireless communications [4], in which it is expected to play a central role in IoT [5].

In the problem of joint deconvolution and demixing [6], the goal is to reconstruct the signals $x_{s}$ and kernels $w_{s}$ from

$$
y=\sum_{s \in[S]} x_{s} \circledast w_{s},
$$

extending blind deconvolution to a sum of convolutions. Like the classic blind deconvolution problem [7], this problem is ambiguous without further constraints on the signals and kernels (more on the ambiguities of one dimensional blind deconvolution can be found in [8], [9]). Common assumptions include peakiness (e.g., [10], [11]), sparsity (e.g., [12], [13]), and subspace priors (e.g., [14]).

Our problem (1) was solved using convex Nuclear norm minimization, exploiting the rank-1 structure of the lifted problem [6], [15]-[17], assuming the subspace prior suggested by [14]. Probabilistic linear guarantees for the relationship between the amount of measurements in $y$, and the amount of signal-kernel pairs, $S$, were derived for this method [17].

The convex Nuclear minimization approach allows the derivation of theoretical guarantees with minimal assumptions, though combined with the common lifting procedure it might result in high computational complexity. Non-convex approaches were also explored in the context of blind deconvolution [18] and demixing [19]-[24], with significant lower computation time. Though the theoretical result in [18] is in line with those achieved in the convex approach, the expansions to demixing via the non-convex methods are still

(C)2021 IEEE. Personal use of this material is permitted. Permission from IEEE must be obtained for all other uses, in any current or future media including reprinting/republishing this material for advertising or promotional purposes, creating new collective works, for resale or redistribution to servers or lists, or reuse of any copyrighted component of this work in other works. with quadratic guarantees. A thorough review of nonconvex algorithms in a broader context of general matrix completion problems can be found in [25].

A related variant of (1) considers a scenario where $S$ sources transmit signals to $R$ receivers. Each path is modeled by a different, unknown convolution kernel, yielding

$$
y_{r}=\sum_{s \in[S]} x_{s} \circledast w_{r s}, \quad r \in[R],
$$

where $y_{r}$ is the measurement of the $r$ th out of $R$ receivers. This is equivalent to the blind MIMO model presented in [26]. Their method also uses Nuclear norm minimization employing the rank-1 structure of the lifted problem, assuming that the signals reside in a known subspace. This leads to an optimization problem on a matrix consisting of rank-1 blocks, which do not share variables. However, the solution cannot be obtained separately for each block and requires solving the full optimization problem of dimension $R K \times S N$, where $K, N$ are the dimensions of the signals and kernels subspaces.

Contribution. This work assumes that all signals lay in the same subspace (as do all kernels). This allows solving separate rank- $S$ problems instead of a single, large rank-1 problem, which reduces the computational complexity significantly. We show linear performance in the reconstruction of each rank$S$ matrix and develop theoretical guarantees that match those of the rank-1 case [17]. The advantage of our solution is its improved computational complexity; Instead of a single large problem, we solve a few small optimization problems with better variables to degrees of freedom ratio. See Section III

Given the recovered rank- $S$ matrix, the standard form to retrieve the originating vectors from it is by SVD [14]. However, in the rank-S case, there is an ambiguity of the spanning base, i.e., the singular vectors are generally not the originating vectors. To overcome this, we suggest an algorithm that uses the fact that the signals are shared between all receivers, and can reconstruct all signals and kernels under some normalization assumptions, to be described in Section III-B

Notations. Unless stated otherwise, we use the following norm notations throughout our derivations. $\|\cdot\|$ without subscript is the operator norm of the appropriate subject: $\|\mathcal{A}\|=\|\mathcal{A}\|_{F \rightarrow 2}$ for sampling operators; $\|A\|=\|A\|_{2 \rightarrow 2}$ for matrices. We use $[S]$ to describe the set of integers $1, \ldots, S$. $x_{s} / y_{r s}$ denotes the $s$ column of a matrix $X / Y_{r}$. To denote an inequality up to a constant depending on $\omega$ we use $\lesssim \omega$.

\section{THE PROBLEM SETUP}

Consider that $R$ convolutions-sums are measured, sharing the signals while the kernels are different per source-receiver path. Let the convolution kernel matrix for each sensor be 
$W_{r} \in \mathbb{C}^{L \times S}$, and let $X \in \mathbb{C}^{L \times S}$ be $S$ signals of length $L$. Assume that the signals and kernels reside in a low $(N, K)$ dimensional subspaces spanned by the columns of the known matrices $B, C$, such that

$$
\begin{array}{cc}
X=C M, & M \in \mathbb{C}^{N \times S}, \\
W_{r}=B H_{r}, & H_{r} \in \mathbb{C}^{K \times S},
\end{array}
$$

where $B \in \mathbb{C}^{L \times K}$ is assumed to have orthogonal columns, while the entries of $C \in \mathbb{C}^{L \times N}$ are independent and follow a standard circular-symmetric normal distribution. The standard subspace prior [14] can be achieved in our framework with an appropriate choice of coding matrices. Therefore, our assumption is reasonable and holds no limitation to the common approach. Denote the "column-wise" cyclic convolution of $X$ and $W_{r}$ as $X \circledast W_{r}=\tilde{Y}_{r} \in \mathbb{C}^{L \times S}$. Then the measurement of each sensor reads as

$$
y_{r}=\sum_{s} x_{s} \circledast w_{r s}+e=\sum_{s} \tilde{y}_{r s}+e, \quad s \in[S],
$$

where $e$ is additive noise. Let $F$ be the $L$ dimensional DFT matrix, $\operatorname{diag}\left(f_{l}\right)$ the diagonal matrix consisting of the $l$ th column of $F, f_{l}$, and $A_{l}=\sqrt{L} B^{*} F^{*} \operatorname{diag}\left(f_{l}\right) \bar{F} \bar{C}$. Then for a given signal-kernel pair, the $l$ th measurement component in Fourier domain is given by [27]

$$
\hat{\tilde{y}}_{r s l} \triangleq\left(F\left(x_{s} \circledast w_{r s}\right)\right)_{l}=\left\langle A_{l}, m_{s} h_{r s}^{*}\right\rangle,
$$

Thus, the $l$ th Fourier entry of the signal-kernel pairs sum is

$$
\begin{aligned}
\hat{y}_{r l} & \triangleq\left(F\left(y_{r}\right)\right)_{l}=\sum_{s} \hat{\tilde{y}}_{r s l}+\hat{e}_{l}=\sum_{s}\left\langle A_{l}, m_{s} h_{r s}^{*}\right\rangle+\hat{e}_{l} \\
& =\left\langle A_{l}, \sum_{s} m_{s} h_{r s}^{*}\right\rangle+\hat{e}_{l}=\left\langle A_{l}, M H_{r}^{*}\right\rangle+\hat{e}_{l} .
\end{aligned}
$$

The complete linear measurement operator $\mathcal{A}: \mathbb{C}^{N \times K} \rightarrow \mathbb{C}^{L}$ in Fourier domain is therefore defined by

$$
\mathcal{A}(\cdot)=\left[\left\langle A_{1}, \cdot\right\rangle, \ldots,\left\langle A_{L}, \cdot\right\rangle\right]^{T},
$$

which leads to writing the measured vector at receiver $r$ as

$$
\hat{y}_{r}=\mathcal{A}\left(M H_{r}^{*}\right)+\hat{e}=\mathcal{A}\left(Z_{r}\right)+\hat{e},
$$

where $Z_{r} \triangleq M H_{r}^{*}$. The problem of demixing convolved signals and kernels is hence the reconstruction of the signals subspace coefficients vectors $M$ and the convolution kernels subspace coefficients vectors $H_{r}$ from the Fourier transform of the measurement vector $y_{r}$.

\section{SEPARABLE OPTIMIZATION FOR SVD BASED JOINT DECONVOLUTION AND DEMIXING}

Casting the problem as a matrix recovery problem, as in (9), allows the use of rank minimization algorithms since $Z_{r}$ is known to be a rank- $S$ matrix. Yet, unlike the rank-1 case, the recovery of the matrix is insufficient for the reconstruction of the actual signals and kernels. As we further explain hereafter, this is due to a wider ambiguity in the factorization of the matrix, that is not resolved in the SVD process, which is the standard tool for vector recovery in the rank-1 case [14].

Our framework considers a model in which $S$ sources transmit signals to $R$ receivers, while each channel is represented by a different convolution kernel. Thus, each receiver measures the mixture of $S$ convolutions, $y_{r}$. Our method has two stages: 1. Matrix recovery: reconstruct $Z_{r}$ from $y_{r}$ via Nuclear norm minimization at each receiver, separately.

2. Vector recovery: estimate $M, H_{r}$ from $Z_{r}=M H_{r}^{*}$. This step uses the estimated $Z_{r}$ from all receivers and requires solving a quadratic equation system of $S^{2}$ variables, regardless of $L, K$, and $N$, which may be generally much larger.

\section{A. Matrix recovery}

Assume that all signals and kernels have the same coding matrix. Thus, for each receiver, we may recover $Z_{r} \in \mathbb{C}^{K \times N}$, which has rank $\mathbf{S}$, by solving

$$
\min _{Z}\|Z\|_{*} \quad \text { s.t. } \quad\left\|\mathcal{A}(Z)-\hat{y}_{r}\right\|_{2} \leq \tau .
$$

Computationally, this is equivalent to the standard convex approach to blind deconvolution, with no demixing [14].

For comparison, [17] deal only with the case of one receiver $(R=1)$ so the total degrees of freedom (DoF) are $S(K+N)$. In the noiseless case, they solve an optimization problem for $S$ rank-1 matrices, i.e. with $S K N$ variables:

$$
\min _{Z_{s}} \sum_{s}\left\|Z_{s}\right\|_{*} \quad \text { s.t. } \quad\left\|\sum_{s} \mathcal{A}_{s}\left(Z_{s}\right)-\hat{y}\right\|_{2} \leq \tau .
$$

Compared to this approach, we have more measurements per signal $(R \geq S)$; This trades off the number of measurements and the ability to solve smaller problems.

Ahmed [26] presented a convex approach to blind MIMO, which is more similar to our case in the sense that he too has multiple receivers. Yet, he solves the problem directly (i.e. with rank one blocks) so he may have $R<S$. When $R=1$, this is exactly (11). When $R \geq S$, this is equivalent to our problem. It has $S N+R S K$ degrees of freedom ( $S$ signals of length $N$ and $S$ kernels of length $K$ in each of the $R$ receivers, without the normalization implications). He solves a similar problem to the former case, only for a sum of $S$ rank-1 matrices of size $R K \times N$, meaning with $S R K N$ variables. As they also note, although the different matrices in the sum share no common variables, the problem cannot be separated.

In our setup we use the shared information to be able to separate the problems, but then the information is no longer shared and there is no sample complexity gain. The "full" approach of the same scheme [26], is on the other side of the trade-off - they show better sample complexity for more receivers but solve a bigger problem. Note though that they show it only empirically, where the dependency decreases quite quickly with the increase in $R$. They also conjecture a linear sample complexity bound (which is what we formally prove). It would be interesting to think of an approach in the middle of the trade-off, where we use our shared subspace assumption in a way that has provably better sample complexity. We leave this to future work.

Table II summarizes the complexity of each method. For each of the methods mentioned above, we detail the desired DoFs, the actual number of variables in the problem, the number of sub-problems ("\#Prob"), extra optimization variables ("ext. op."), the total number of variables, and finally the ratio 
TABLE I: Complexity comparison of the different methods. \#Prob. is the number of separate problems in each work.

\begin{tabular}{|c|c|c|c|}
\hline Method & {$[17]$} & {$[26$} & Ours \\
\hline \hline DoF & $S(K+N)$ & $S(R K+N)$ & $S(R K+N)$ \\
\hline $\begin{array}{c}\text { Opt. } \\
\text { vars }\end{array}$ & $S K N$ & $S R K N$ & $K N$ \\
\hline \#Prob. & 1 & 1 & $R$ \\
\hline ext. op. & - & - & $S^{2}$ \\
\hline Total & $S K N$ & $S R K N$ & $R \times K N+S^{2}$ \\
\hline Ratio & $\frac{K N}{K+N}$ & $\frac{R K N}{R K+N}$ & $\frac{R K N+S^{2}}{S(R K+N)}$ \\
\hline R & $R=1$ & $R \geq 1$ & $R \geq S$ \\
\hline
\end{tabular}

between the optimization variables and the desired DoF. The bottom line includes the range of receivers regime handled by each method. Notice that we have fewer optimization variables, as we keep our matrices small. Moreover, we also have a better (lower) ratio between the optimization variables and the degrees of freedom.

\section{B. Vector recovery: find basis transformation}

Once $Z_{r}$ is restored, we use its SVD decomposition to reconstruct the originating vectors (the signals and kernels coefficients). Thus $Z_{r}=U \Lambda V^{*} \triangleq \tilde{M}_{r} \tilde{H}_{r}^{*}$, where $\tilde{m}_{r i}=$ $\sqrt{\Lambda_{i i}} u_{i}$ (similarly for $\tilde{h}_{r i}$ ). In the rank-1 case, this is trivial: $Z_{r}=m h_{r}^{*}=\tilde{m}_{r 1} \sigma \sigma^{-1} \tilde{h}_{r 1}^{*}$ for any $\sigma \neq 0$. Thus, the estimated vectors are $m=\tilde{m}_{r 1}, h_{r}=\tilde{h}_{r 1}$ up to scale and sign. These ambiguities of the rank-1 case become an ambiguity of spanning base in higher ranks, which can be expressed by

$$
M H_{r}^{*}=\tilde{M}_{r} T^{r}\left(T^{r}\right)^{-1} \tilde{H}_{r}^{*},
$$

where $T^{r} \in \mathbb{C}^{S \times S}$ is the basis transformation from the columns of $\tilde{M}_{r}$ to the columns of the original $M$.

To restore the original vector pairs, we measure $R$ convolutions-sums, assuming that $M$ is constant in all of them while only $H_{r}$ is changing. This corresponds to the blind MIMO scheme in [26] and allows us to pose enough constraints to determine $T^{r}, \forall r \in[R]$, as follows: To exploit the fact that $M$ is shared, we want to express the relation in (12) with the same transformation matrix $T$, for all $r \in[R]$. Thus, we choose an arbitrary $r_{0} \in[R]$. The relation between the transformation matrices at different receivers is given by

$$
T^{r}=\left(T^{r r_{0}}\right)^{-1} T^{r_{0}},
$$

where $T^{r r_{0}} \triangleq \tilde{M}_{r_{0}}^{+} \tilde{M}_{r}$ and ${ }^{+}$is the pseudo-inverse. Note that we can always invert $\tilde{M}_{r_{0}}^{*} \tilde{M}_{r_{0}}$ as $\tilde{M}_{r_{0}}$ has orthogonal columns (due to SVD). To recover the original coefficients, it is sufficient to find the $S^{2}$ entries of $T^{r_{0}}$. Note that it satisfies

$$
\begin{aligned}
M & =\overbrace{\tilde{M}_{r}\left(T^{r r_{0}}\right)^{-1}}^{\tilde{M}_{r_{0}}} T^{r_{0}}, \\
H_{r} & =\underbrace{\tilde{H}_{r}\left(T^{r r_{0}}\right)^{*}}_{\triangleq G_{r}}\left(T^{r_{0}}\right)^{-*} .
\end{aligned}
$$

This equations system allows adding constraints on the same variable basis transformation matrix, $T^{r_{0}}$, by increasing $R$. Note that we do not know $M$ and $H_{r}$. Thus, we need to add some constraints on them to be able to recover $T^{r_{0}}$.
One-sided constraints. Assuming normalized kernel coefficients (a standard assumption due to the scale ambiguity of rank-1 case) and considering (14) leads to the equations

$$
\left\|h_{r s}\right\|^{2}=1=\left(\left(T^{r_{0}}\right)_{s}^{-*}\right)^{*} G_{r}^{*} G_{r}\left(T^{r_{0}}\right)_{s}^{-*}, r \in[R],
$$

where $T_{s}^{r_{0}}$ is the sth column of $T^{r_{0}}$. Having the original coefficients vectors equally normalized means that the equation system is column separable w.r.t $T^{r_{0}}$. Thus, we get $R$ equations for the $S$ variables of $T_{s}^{r_{0}}$ for any $s \in[S]$.

Posing $l_{2}$ norm constraints on the original signals or kernels coefficients leads to a system of quadratic equations, which has an exponential number of solutions even in the fully determined case; A fully determined system with $S$ equations and $S$ variables lead to $2^{S}$ solutions. When $R=S$, the system has at least $2^{S-1}$ solutions (we are agnostic to global phase ambiguity). While this might suffice when $S=2$, it is insufficient for more signal-kernel pairs. Empirically we observed that solving this equation system by optimization for the entire matrix $T^{r_{0}}$, i.e., simultaneously solving for all columns of $T^{r_{0}}$, produces only the $S$ correct results even for $R=S+1$, despite the exponential amount of valid solutions. This was resolved using the Matlab non-linear equation solver fsolve with the trust region Dogleg algorithm.

Two-sided constraints. Posing the normalization constraints on both $M$ and $H_{r}$, and using it with (14) and (15), leads to the equation system

$$
\begin{aligned}
\left\|m_{s}\right\|^{2} & =1=\left(T_{s}^{r_{0}}\right)^{*} \tilde{M}_{r_{0}}^{*} \tilde{M}_{r_{0}} T_{s}^{r_{0}}, \\
\left\|h_{r s}\right\|^{2} & =1=\left(\left(T^{r_{0}}\right)_{s}^{-*}\right)^{*} G_{r}^{*} G_{r}\left(T^{r_{0}}\right)_{s}^{-*} .
\end{aligned}
$$

This adds an additional constraint. However, although each subproblem $([17),(18)$ ) is column separable, the total system is not, as the variables in each of the separate sets are the adjugate matrices of one another (up to the determinant factor). In fact, we are looking for the correct set of $S$ solutions, out of $\left(\begin{array}{c}2^{S-1} \\ S\end{array}\right)$ possible column sets that can solve 17 , which can be jointly inverted to the correct set of solutions of (18). This setup appears to impose "hidden" constraints. We conjecture that they resolve the ambiguity of the quadratic equations for $R=S$, as we empirically show in Section $\mathrm{V}$.

\section{Matrix recovery guarantees}

We turn now to provide theoretical guarantees for the recovery of $Z_{r}$. Jung et al. [17] were the first to present a linear guarantee for the uniqueness of recovery when solving [11. Ahmed [26], which presented a convex approach to blind MIMO, conjectured that also in his case $L$ is linear w.r.t. $\max (R, S)$, without proof. Such guarantees [6], [14], [17] rely on the fact that the retrieved matrix is rank-1, and, thus, do not apply in our case. The following provides a guarantee for the reconstruction of higher rank matrices (by solving (10), with the same assumptions on $B, C$ and a similar linear result.

Theorem 1. Let $\omega \geq 1$ and let $y \in \mathbb{C}^{L}$ be given by (5), with $\|e\|_{2} \leq \tau$. Assume that

$$
L \gtrsim_{\omega} S\left(K \mu^{2} \log \left(K \mu^{2}\right)+N \mu_{H}^{2}\right) \log ^{3} L,
$$


then with probability of at least $1-\mathcal{O}\left(L^{-\omega}\right)$ the minimizer $\hat{X}$ of (10) satisfies

$$
\left\|\hat{X}-X_{0}\right\|_{F} \lesssim \omega \tau \sqrt{S \max \left\{1, \frac{S K \mu^{2} N}{L}\right\} \log ^{3}(L)} .
$$

Compared to [17], we have the same lower bound on $L$ but effectively more measurements per signal (all $y$-vectors). This is part of the tradeoff that enables us to have smaller computational problems, as discussed in Section III-A.

\section{PRoOF OF THE MAIN THEOREM}

The structure of our proof is similar to the ones in [6], [14], [17]. We prove sufficient conditions for recovery, assuming the existence of an inexact dual certificate: We show that our measurement operator fulfills a Local Isometry Property (see (23)) on the relevant spaces (those defined in Def. 2 and 4), and then construct the dual certificate using the Golfing Scheme.

\section{A. Preliminary Definitions}

We start with preliminary definitions. The sgn function is defined in the functional sense, i.e.

$$
\operatorname{sgn}(A)=U \operatorname{diag}\left(\operatorname{sgn}\left(\sigma_{1}\right), \ldots, \operatorname{sgn}\left(\sigma_{r}\right)\right) V^{*},
$$

where $A=U \Sigma V^{*}$ is the SVD of $A$ and $\left\{\sigma_{i}\right\}$ are the singular values of $A$. This differs from the definition in [17] and is important for proving the results for the reconstruction of matrices with rank exceeding one. We denote by $\hat{H} \Lambda \hat{M}^{*}$ the SVD decomposition of $X_{0}=H M^{*}$, and define

$$
\mathcal{M}=\left\{Z \mid Z \in \mathbb{C}^{K \times N}\right\},
$$

to be the space of matrices of the appropriate size.

Solution space. We now turn to define the solution space. (Note that some other works refer to it as the tangent space.)

Definition 2 (Solution space). Let $\hat{H} \Lambda \hat{M}^{*}$ be the SVD decomposition of $X_{0}=H M^{*}$. Given

$$
\mathcal{T}_{M}=\left\{V \hat{M}^{*} \mid V \in \mathbb{C}^{K \times S}\right\}, \mathcal{T}_{H}=\left\{\hat{H} U^{*} \mid U \in \mathbb{C}^{N \times S}\right\},
$$

the solution space is defined as $\mathcal{T}=\mathcal{T}_{M}+\mathcal{T}_{H}$.

Local Isometry Property (LIP). An operator $\mathcal{A}$ satisfies the LIP with a constant $\delta$ if $\forall X \in \mathcal{T}$

$$
(1-\delta)\|X\|_{F}^{2} \leq\|\mathcal{A}(X)\|_{2}^{2} \leq(1+\delta)\|X\|_{F}^{2} .
$$

Partition of measurements and incoherence. In our proofs, we also use extended spaces that are slightly larger than the solution space. These spaces are induced by the measurements partitioning, required for the Golfing scheme [28]. Define the kernels subspace matrix coherence parameter

$$
\mu^{2}=\frac{L}{K} \max _{l \in[L]}\left\|b_{l}\right\|_{2}^{2}
$$

where $b_{l}$ is the $l$ th column of $B^{T}$. Notice that $1 \leq \mu^{2} \leq \frac{L}{K}$.

Using the Golfing Scheme [28] requires a division of the $L$ measurements into $P$ non-overlapping sets. We denote the indexing of each set by $\Gamma_{p}$ where $p \in[P]$. Thus, $\cup_{p} \Gamma_{p}=[L]$. Each set is associated with its linear measurement operator

$$
\mathcal{A}^{p}(Z)=\left\{\left\langle A_{l}, Z\right\rangle\right\}_{l \in \Gamma_{p}}
$$

For convenience of writing, we define

$$
\begin{gathered}
T_{p}=\frac{L}{Q} \sum_{l \in \Gamma_{P}} b_{l} b_{l}^{*} \\
S_{p}=T_{p}^{-1}=\left(\frac{L}{Q} \sum_{l \in \Gamma_{P}} b_{l} b_{l}^{*}\right)^{-1}
\end{gathered}
$$

where $Q \triangleq \frac{L}{P}$. To guarantee the convergence of the Golfing Scheme with high probability, the partition must be chosen such that $T_{p} \approx I_{K}$ for all $p \in[P]$. Thus, we require that

$$
\max _{p \in[P]}\left\|T_{p}-I\right\| \leq \nu
$$

for a small enough $\nu$. Moreover, the partition needs to be $\omega$ admissible in the following sense.

Definition 3. Let $\omega \geq 1$ and let $\left\{\Gamma_{p}\right\}_{p \in P}$ be a partition of $[L]$. The set $\left\{\Gamma_{p}\right\}_{p \in P}$ is said to be $\omega$-admissible if the following conditions are satisfied:

1) $\frac{1}{2} Q \leq\left|\Gamma_{p}\right| \leq \frac{3}{2} Q$ for all $p \in P$, where $Q=\frac{L}{P}$

2) 28 is fulfilled with $\nu=\frac{1}{32}$

3) $\frac{1}{2} \log (8 \tilde{\gamma} \sqrt{S}) \leq P \leq \log (8 \tilde{\gamma} \sqrt{S})$, where

$$
\tilde{\gamma}=2 \sqrt{\omega \max \left\{1, \frac{S K \mu^{2} N}{L}\right\} \log (L+S K N)} .
$$

The existence of such partition is guaranteed by Lemma 3 in [17]. For a fixed $\omega$-admissible partition we can define

$$
\mu_{H}^{2}=L \max \left\{\max _{l \in L, s \in S}\left|b_{l}^{*} h_{s}\right|^{2}, \max _{l \in L, s \in S, p \in P}\left|b_{l}^{*} S_{p} h_{s}\right|^{2}\right\},
$$

where $1 \leq \mu_{H}^{2} \leq\left(\frac{32}{31}\right)^{2} K \mu^{2} \lesssim L$. Now we can define the extended solution space for each $p$.

Definition 4 (Extended solution space). Fix $p \in[P]$. The extended solution space is defined as $\mathcal{T}^{p}=\mathcal{T}+\mathcal{T}_{S^{p} H}$, where

$$
\mathcal{T}_{S^{p} H}=\left\{S_{p} \hat{H} U^{*} \mid U \in \mathbb{C}^{N \times S}\right\} .
$$

Orthogonal projection operators. We can define the orthogonal projection operator onto the solution space $\mathcal{P}_{\mathcal{T}}$ by

$$
\mathcal{P}_{\mathcal{T}}(Z)=\mathcal{P}_{\hat{H}} Z+Z \mathcal{P}_{\hat{M}}-\mathcal{P}_{\hat{H}} Z \mathcal{P}_{\hat{M}}
$$

and the orthogonal projection operator onto the complementary space $\mathcal{T}^{\perp}$ by

$$
\mathcal{P}_{\mathcal{T}^{\perp}}(Z)=\left(I-\mathcal{P}_{\mathcal{T}}\right) Z=\left(I_{K}-\mathcal{P}_{\hat{H}}\right) Z\left(I_{N}-\mathcal{P}_{\hat{M}}\right)
$$

where

$$
\begin{aligned}
& \mathcal{P}_{\hat{H}}=\hat{H} \hat{H}^{*}, \mathcal{P}_{\hat{M}}=\hat{M} \hat{M}^{*} \\
& \mathcal{T}^{\perp}=\operatorname{span}\left\{v u^{*} \mid u \perp\left\{h_{s}\right\}_{s \in[S]}, v \perp\left\{m_{s}\right\}_{s \in[S]}\right\} .
\end{aligned}
$$




\section{B. Sufficient conditions for recovery}

We first find sufficient conditions for recovery in the presence of noise.

Lemma 5. Suppose that $\mathcal{A}$ satisfies the $\delta$-local isometry property on $\mathcal{T}$ and set $\gamma=\|\mathcal{A}\|$. Furthermore, suppose that there is $Y=\mathcal{A}^{*} z$ for some $z \in \mathbb{C}^{L}$ such that

$$
\begin{gathered}
\left\|\mathcal{P}_{\mathcal{T}}(Y)-\operatorname{sgn}\left(X_{0}\right)\right\|_{F} \leq \alpha \\
\left\|\mathcal{P}_{\mathcal{T}^{\perp}}(Y)\right\| \leq \beta
\end{gathered}
$$

where $\alpha, \beta \geq 0$ are constants such that $1-\beta-\frac{\alpha \gamma}{\sqrt{1-\delta}} \geq \frac{1}{2}$, $\alpha \leq 1$ and $\sqrt{1-\delta} \geq \frac{1}{2}$. If $\hat{X}$ is a minimizer of

$$
\min _{X}\|X\|_{*} \quad \text { s.t } \quad\|\mathcal{A}(X)-\hat{y}\|_{2} \leq \tau,
$$

then

$$
\left\|\hat{X}-X_{0}\right\|_{F} \lesssim \tau(1+\gamma)\left(1+\|z\|_{2}\right) .
$$

Proof. Set $V=\hat{X}-X_{0}$. We want to bound $\|V\|_{F} \leq$ $\left\|\mathcal{P}_{\mathcal{T}}(V)\right\|_{F}+\left\|\mathcal{P}_{\mathcal{T}^{\perp}}(V)\right\|_{F}$. Since $\hat{X}$ is the minimizer of 37] we have

$$
\|\mathcal{A}(V)\|_{2} \leq\|\mathcal{A}(\hat{X})-\hat{y}\|_{2}+\left\|\hat{y}-\mathcal{A}\left(X_{0}\right)\right\|_{2} \leq 2 \tau .
$$

Combined with the local isometry property $(23), \gamma$ being the operator norm of $\mathcal{A}$ and the triangle inequality we get

$$
\begin{aligned}
\left\|\mathcal{P}_{\mathcal{T}}(V)\right\|_{F} & \leq \frac{1}{\sqrt{1-\delta}}\left\|\mathcal{A}\left(\mathcal{P}_{\mathcal{T}}(V)\right)\right\|_{2} \\
& \leq \frac{1}{\sqrt{1-\delta}}\left(\left\|\mathcal{A}\left(\mathcal{P}_{\mathcal{T}^{\perp}}(V)\right)\right\|_{2}+\|\mathcal{A}(V)\|_{2}\right) \\
& \left.\leq \frac{1}{\sqrt{1-\delta}}\left(\gamma \| \mathcal{P}_{\mathcal{T}^{\perp}}(V)\right) \|_{2}+2 \tau\right) .
\end{aligned}
$$

To upper bound $\left\|\mathcal{P}_{\mathcal{T}^{\perp}}(V)\right\|_{2}$, we choose $Z_{0} \in \mathcal{T}^{\perp}$ such that $\left\|Z_{0}\right\| \leq 1-\beta$ and $\left\langle Z_{0}, V\right\rangle_{F}=(1-\beta)\left\|\mathcal{P}_{\mathcal{T}^{\perp}}(V)\right\|_{*}$. This is possible due to the duality of the norms $\|\cdot\|_{2 \rightarrow 2}$ and $\|\cdot\|_{*}$. Note that $\left\|\operatorname{sgn}\left(X_{0}\right)+\mathcal{P}_{\mathcal{T}^{\perp}}(Y)+Z_{0}\right\| \leq 1$ since $\operatorname{sgn}\left(X_{0}\right) \perp \mathcal{P}_{\mathcal{T} \perp}(Y)+Z_{0}$, the mentioned bound on $\left\|Z_{0}\right\|$, (36) and $\left\|\operatorname{sgn}\left(X_{0}\right)\right\| \leq 1$. Using this duality again, we get

$$
\begin{aligned}
& \left\|X_{0}+V\right\|_{*}=\sup _{Z \in \mathbb{C}^{K \times N},\|Z\| \leq 1}\left|\left\langle Z, X_{0}+V\right\rangle_{F}\right| \\
& \geq \operatorname{Re}\left(\left\langle\operatorname{sgn}\left(X_{0}\right)+\mathcal{P}_{\mathcal{T}^{\perp}}(Y)+Z_{0}, X_{0}+V\right\rangle_{F}\right) \\
& =\left\|X_{0}\right\|_{*}+\operatorname{Re}\left(\left\langle\mathcal{P}_{\mathcal{T}^{\perp}}(Y)+Z_{0}, X_{0}\right\rangle_{F}\right)+ \\
& \operatorname{Re}\left(\left\langle\operatorname{sgn}\left(X_{0}\right)+\mathcal{P}_{\mathcal{T}^{\perp}}(Y), V\right\rangle_{F}\right)+\operatorname{Re}\left(\left\langle Z_{0}, V\right\rangle_{F}\right) \\
& =\left\|X_{0}\right\|_{*}+\operatorname{Re}\left(\left\langle\operatorname{sgn}\left(X_{0}\right)+\mathcal{P}_{\mathcal{T}^{\perp}}(Y), V\right\rangle_{F}\right)+ \\
& (1-\beta)\left\|\mathcal{P}_{\mathcal{T}^{\perp}}(Y)\right\|_{*}=\left\|X_{0}\right\|_{*}+(1-\beta)\left\|\mathcal{P}_{\mathcal{T}^{\perp}}(V)\right\|_{*}+ \\
& \operatorname{Re}\left(\left\langle\operatorname{sgn}\left(X_{0}\right)-\mathcal{P}_{\mathcal{T}}(Y), V\right\rangle_{F}+\langle Y, V\rangle_{F}\right),
\end{aligned}
$$

where the first equality is due to $\|X\|_{*}=\langle\operatorname{sgn}(X), X\rangle_{F}$ (see 210$)$ and the third equality follows $\operatorname{Re}\left(\left\langle\mathcal{P}_{\mathcal{T} \perp}(V)+\right.\right.$ $\left.\left.Z_{0}, X_{0}\right\rangle_{F}\right)=0$. Notice, that our definition of the sign function in [21], which differs from the one in [17], is essential for this step. The last step is due to $Y=\mathcal{P}_{\mathcal{T}}(Y)+\mathcal{P}_{\mathcal{T} \perp}(Y)$.
We now examine the term $\operatorname{Re}\left(\left\langle\operatorname{sgn}\left(X_{0}\right)-\mathcal{P}_{\mathcal{T}}(Y), V\right\rangle_{F}\right)$ in the last line of 41). By Cauchy-Schwarz, the upper bound for $\left\|\mathcal{P}_{\mathcal{T}}(V)\right\|_{F}$ in (40) and the assumption in 35), we get

$$
\begin{aligned}
& \operatorname{Re}\left(\left\langle\operatorname{sgn}\left(X_{0}\right)-\mathcal{P}_{\mathcal{T}}(Y), V\right\rangle_{F}\right) \\
& \geq-\left\|\operatorname{sgn}\left(X_{0}\right)-\mathcal{P}_{\mathcal{T}}(Y)\right\|_{F}\left\|\mathcal{P}_{\mathcal{T}}(V)\right\|_{F} \\
& \left.\geq \frac{-\alpha}{\sqrt{1-\delta}}\left(\gamma \| \mathcal{P}_{\mathcal{T}^{\perp}}(V)\right) \|_{2}+2 \tau\right)
\end{aligned}
$$

where in the first inequality we have also used the fact that $\operatorname{sgn}\left(X_{0}\right)-\mathcal{P}_{\mathcal{T}}(Y) \in \mathcal{T}$. To bound the term $\operatorname{Re}\left(\langle Y, V\rangle_{F}\right)$ in the last line of (41), note that by Cauchy-Schwarz and (39),

$$
\begin{aligned}
\operatorname{Re}\left(\langle Y, V\rangle_{F}\right) & =\operatorname{Re}\left(\left\langle\mathcal{A}^{*}(z), V\right\rangle_{F}\right) \\
& =\operatorname{Re}\left(\langle z, \mathcal{A}(V)\rangle_{2}\right) \geq-2\|z\|_{2} \tau
\end{aligned}
$$

Putting (42) and 43) back into 41, we get

$$
\begin{aligned}
\|\hat{X}\|_{*} & =\left\|X_{0}+V\right\|_{*} \\
& \geq\left\|X_{0}\right\|_{*}+\left(1-\beta-\frac{\alpha \gamma}{\sqrt{1-\delta}}\right)\left\|\mathcal{P}_{\mathcal{T}^{\perp}}(V)\right\|_{*} \\
& -2 \tau\left(\|z\|_{2}+\frac{\alpha}{\sqrt{1-\delta}}\right) .
\end{aligned}
$$

Since $\hat{X}$ is the minimizer of the Nuclear norm, we have $\|\hat{X}\|_{*} \leq\left\|X_{0}\right\|_{*}$ and therefore

$$
\left\|\mathcal{P}_{\mathcal{T}^{\perp}}(V)\right\|_{*} \leq \frac{2 \tau\left(\|z\|_{2}+\frac{\alpha}{\sqrt{1-\delta}}\right)}{\left(1-\beta-\frac{\alpha \gamma}{\sqrt{1-\delta}}\right)} .
$$

Considering our assumptions on the constants we get $\left\|\mathcal{P}_{\mathcal{T} \perp}(V)\right\|_{F} \lesssim \tau\left(\|z\|_{2}+1\right)$. Finally, we can bound

$$
\begin{gathered}
\|V\|_{F} \leq\left\|\mathcal{P}_{\mathcal{T}}(V)\right\|_{F}+\left\|\mathcal{P}_{\mathcal{T}^{\perp}}(V)\right\|_{F} \\
\lesssim(1+\gamma)\left\|\mathcal{P}_{\mathcal{T}^{\perp}}(V)\right\|_{F}+\tau \lesssim \tau(1+\gamma)\left(\|z\|_{2}+1\right)
\end{gathered}
$$

The error bound requires us to bound also $\gamma$, the operator norm of the measurement operator $\mathcal{A}$. This is done in the following lemma, with its proof in App. B-A

Lemma 6 (Operator norm bound). Let $\omega \geq 1$. Then with probability of at least $1-2 L^{-\omega}$,

$$
\|\mathcal{A}\| \leq 2 \max \left\{1, \sqrt{\frac{N K}{L}} \mu\right\} \sqrt{\log (L+S K N)}
$$

\section{Local Isometry Property}

We now show that the measurement operators $\mathcal{A}, \mathcal{A}^{p}(8)$, (25) act as approximate isometries on $\mathcal{T}, \mathcal{T}^{p}$ (Def. 2, 47.

Theorem 7. Fix $\omega \geq 1$. Suppose that

$$
Q \geq C_{\omega} \delta^{-2} S\left(K \mu^{2} \log (L) \log ^{2}\left(K \mu^{2}\right)+N \mu_{H}^{2}\right),
$$

then with probability $1-\mathcal{O}\left(L^{-\omega}\right)$ the operator $\mathcal{A}$ satisfies (23) (LIP), and for all $p \in[P]$, every $Y \in \mathcal{T}^{p}=\mathcal{T}+\mathcal{T}_{S^{p} H}$ fulfills

$$
(1-\delta)\left\|T_{p}^{\frac{1}{2}} Y\right\|_{F}^{2} \leq \frac{L}{Q}\left\|\mathcal{A}^{p}(Y)\right\|_{2}^{2} \leq(1+\delta)\left\|T_{p}^{\frac{1}{2}} Y\right\|_{F}^{2},
$$


where $T_{p}^{1 / 2}$ denotes the unique positive, self-adjoint matrix whose square is equal to $T_{p}$.

To prove this we need to define the following norms.

Definition 8. For any vector $z \in \mathbb{C}^{K}$ and matrix $Z \in \mathbb{C}^{K \times N}$ :

$$
\|z\|_{B}=\sqrt{L} \max _{l \in[L]}\left|z^{*} b_{l}\right|,\|Z\|_{B}=\sqrt{L} \max _{l \in[L]}\left\|Z^{*} b_{l}\right\|_{2} \cdot
$$

Our strategy is to use the following proposition, proven in App. A and based on Th. 16 regarding suprema of chaos processes. This involves the $\gamma_{2}$ functional, a geometric quantity introduced by Talagrand [29] and defined here in Def. 15, and the distance $d_{B}(\mathcal{Z})=\sup _{Z \in \mathcal{Z}}\|Z\|_{B}$ (similarly for the Frobenius norm). This is further discussed in App. A

Proposition 9. Let $\mathcal{Z} \subset \mathcal{M}$ be a symmetric set and

$$
\begin{gathered}
E=\frac{\gamma_{2}\left(\mathcal{Z},\|\cdot\|_{B}\right)}{\sqrt{Q}}\left(\frac{\gamma_{2}\left(\mathcal{Z},\|\cdot\|_{B}\right)}{\sqrt{Q}}+d_{F}(\mathcal{Z})\right) \\
V=\frac{d_{B}(\mathcal{Z})}{\sqrt{Q}}\left(\frac{\gamma_{2}\left(\mathcal{Z},\|\cdot\|_{B}\right)}{\sqrt{Q}}+d_{F}(\mathcal{Z})\right) \\
U=\frac{1}{Q} d_{B}^{2}(\mathcal{Z})
\end{gathered}
$$

Then for $t \geq 0$ and all $p \in P$,

$$
\begin{gathered}
\mathbb{P}\left(\sup _{Z \in \mathcal{Z}}\left|\frac{L}{Q}\left\|\mathcal{A}^{p}(Z)\right\|_{2}^{2}-\left\|T_{P}^{1 / 2} Z\right\|_{F}^{2}\right| \geq c_{1} E+t\right) \leq \\
2 \exp \left(-c_{2} \min \left(\frac{t^{2}}{V^{2}}, \frac{t}{U}\right)\right) \\
\mathbb{P}\left(\sup _{Z \in \mathcal{Z}}\left|\frac{L}{Q}\|\mathcal{A}(Z)\|_{2}^{2}-\|Z\|_{F}^{2}\right| \geq c_{3} E+t\right) \leq \\
2 \exp \left(-c_{4} \min \left(\frac{t^{2}}{V^{2}}, \frac{t}{U}\right)\right)
\end{gathered}
$$

given that $\left\{\Gamma_{P}\right\}_{p \in P}$ is an $\omega$-admissible partition of $[L]$.

We now apply the proposition on the appropriate sets for proving Th. 7] We define the solution subspaces

$$
\begin{aligned}
\mathcal{B}^{M} & =\left\{X \in \mathcal{T}_{M} \mid\|X\|_{F} \leq 1\right\} \\
\mathcal{B}^{H} & =\left\{X \in \mathcal{T}_{H} \mid\|X\|_{F} \leq 1\right\} \\
\mathcal{B}^{S^{p} H} & =\left\{X \in \mathcal{T}_{S^{p} H} \mid\|X\|_{F} \leq 1\right\} .
\end{aligned}
$$

Note, that these are sets of rank- $S$ matrices. The LIP in Th. 7 follows by applying Proposition 9 on the set (in place of $\mathcal{Z}$ )

$$
\mathcal{W}=\mathcal{B}^{M}+\mathcal{B}^{H}
$$

and in a similar way we get (49) by applying it on the set

$$
\mathcal{W}^{p}=\mathcal{W}+\mathcal{B}^{S^{p} H}
$$

Thus, we only need to estimate the $\gamma_{2}$-functional, $d_{B}(\mathcal{Z})$ and $d_{F}(\mathcal{Z})$ on these sets, which is provided by the following lemma, with its proof in App. B-C

Lemma 10. Suppose that $\mathcal{X}=\mathcal{W}$ or $\mathcal{X}=\mathcal{W}^{p}$ for some $p \in[P]$. Then

$$
\begin{gathered}
d_{F}(\mathcal{X}) \leq 3, \\
d_{B}(\mathcal{X}) \leq 3 \sqrt{K} \mu, \\
\gamma_{2}\left(\mathcal{X},\|\cdot\|_{B}\right) \lesssim \sqrt{S\left(K \mu^{2} \log (L) \log ^{2}\left(K \mu^{2}\right)+N \mu_{H}^{2}\right)} .
\end{gathered}
$$

Now we can prove Th. 7 .

Proof of Th. 7. Fix $p \in[P]$. Using Lemma 10 and choosing the constant $C_{\omega}$ in (48) large enough we get $E \leq \frac{\delta}{2 c_{1}}, V \leq$ $\frac{\delta}{\sqrt{c_{2} \omega \log L}}$ and $U \leq \frac{\delta}{c_{2} \omega \log L}$, where $\mathcal{X} \subset \mathcal{W}^{p}$. The inequality (51) in Proposition 9 with $t=\frac{\delta}{2}$ shows that 49] in Th. 7 holds with probability of $1-\mathcal{O}\left(L^{-\omega}\right)$ (same holds for 52) and (23), with $\mathcal{X} \subset \mathcal{W}$ ). Replacing $\omega$ by $\omega+1$ and using a union bound argument shows that (49) and 23) are satisfied for all $p \in[P]$ with a probability of at least $1-(P+1) \mathcal{O}\left(L^{-\omega-1}\right)=$ $1-\frac{P+1}{L} \mathcal{O}\left(L^{-\omega}\right)=1-\mathcal{O}\left(L^{-\omega}\right)$, which finishes the proof.

\section{Constructing the Dual Certificate.}

We now turn to prove that the assumptions in Lemma 5 hold. As in previous works, we construct the dual certificate via the Golfing Scheme, presented in [28]. Thus, we build the dual certificate with the iterative process

$$
\begin{gathered}
Y_{0}=0, \\
Y_{p}=Y_{p-1}+\frac{L}{Q}\left(\mathcal{A}^{p}\right)^{*} \mathcal{A}^{p} S^{p}\left(\operatorname{sgn}\left(X_{0}\right)-\mathcal{P}_{\mathcal{T}}\left(Y_{p-1}\right)\right),
\end{gathered}
$$

where the final certificate $Y$ is given by

$$
Y=Y_{P}=\sum_{p=1}^{P} \frac{L}{Q}\left(\mathcal{A}^{p}\right)^{*} \mathcal{A}^{p} S^{p} W_{p-1},
$$

and

$$
W_{p} \triangleq \operatorname{sgn}\left(X_{0}\right)-\mathcal{P}_{\mathcal{T}}\left(Y_{p}\right)
$$

First, we show that $Y \in \operatorname{Range}\left(\mathcal{A}^{*}\right)$. Recall that $\mathcal{A}^{p}$ is defined in 25 by taking only the measurements indexed by $l \in \Gamma_{p}$, while having zeros in the other entries. Thus,

$$
\left(\mathcal{A}^{p}\right)^{*} \mathcal{A}^{p} S^{p} W_{p-1}=\mathcal{A}^{*} \mathcal{A}^{p} S^{p} W_{p-1}
$$

and we can write $Y$ as

$$
Y=\mathcal{A}^{*} \sum_{p=1}^{P} \frac{L}{Q} \mathcal{A}^{p} S^{p} W_{p-1},
$$

which is clearly in $\operatorname{Range}\left(\mathcal{A}^{*}\right)$. It thus remains to show that assumptions (35), 36 in Lemma 5 hold.

1) Solution Space Bound: We now show that condition 35 holds. Despite the different definitions and rank, the proof in [17] is adequate also in our case, with very slight modifications. We start with stating a private case of a technical lemma and continue to prove this section's main claim.

Lemma 11 (a private case of Lemma 30 in [17]). Let $\nu \leq \frac{1}{32}$. Then for all $p \in[p]$,

$$
\begin{gathered}
\left\|I-T_{p}^{1 / 2}\right\| \leq \frac{1}{32} \\
\left\|\left(I-S_{p}\right) X\right\|_{F} \leq \frac{1}{31}\|X\|_{F} \\
\left\|S_{p} X\right\|_{F} \leq \frac{32}{31}\|X\|_{F}
\end{gathered}
$$

This allows us to prove the following lemma. 
Lemma 12. Suppose that $\mathcal{A}_{p}$ satisfies the $\delta$-local isometry property on $\mathcal{T}_{p}$ with $\delta=\frac{1}{32}$ for all $p \in[P]$. Then, for all $p \in[P]$,

$$
\left\|W_{p}\right\|_{F} \leq 4^{-p} \sqrt{S}
$$

and, in particular, if $P \geq \frac{1}{2} \log (8 \gamma \sqrt{S})$,

$$
\left\|\operatorname{sgn}\left(X_{0}\right)-\mathcal{P}_{\mathcal{T}}(Y)\right\|_{F} \leq \frac{1}{8 \gamma} .
$$

Proof. By (28) and the triangle inequality we have

$$
(1-\nu)\|X\|_{F} \leq\left\|T_{p}^{1 / 2} X\right\|_{F} \leq(1+\nu)\|X\|_{F} .
$$

Combined with the $\delta$-local isometry property on $\mathcal{T}_{p}$ in 49 ,

$(1-\nu)^{2}(1-\delta)\|X\|_{F}^{2} \leq \frac{L}{Q}\left\|\mathcal{A}^{p}(X)\right\|_{F} \leq(1+\nu)^{2}(1+\delta)\|X\|_{F}^{2}$

for all $X \in \mathcal{T}^{p}$. With $\delta=\nu=\frac{1}{32}$, this implies

$$
\left|\frac{L}{Q}\left\|\mathcal{A}^{p}(X)\right\|_{2}^{2}-\|X\|_{F}^{2}\right| \leq \frac{1}{8}\|X\|_{F}^{2}
$$

for all $X \in \mathcal{T}^{p}$, which is equivalent to

$$
\left\|\mathcal{P}_{\mathcal{T}^{p}}-\frac{L}{Q} \mathcal{P}_{\mathcal{T}^{p}}\left(\mathcal{A}^{p}\right)^{*} \mathcal{A}^{p} \mathcal{P}_{\mathcal{T}^{p}}\right\| \leq \frac{1}{8} .
$$

Notice, that by its definition in 60, we have that

$$
W_{p}=W_{p-1}-\frac{L}{Q} \mathcal{P}_{\mathcal{T}}\left(\mathcal{A}^{p}\right)^{*} \mathcal{A}^{p} S^{p} W_{p-1}
$$

and also that $\left\|W_{p-1}-\mathcal{P}_{\mathcal{T}}(X)\right\|_{F} \leq\left\|W_{p-1}-\mathcal{P}_{\mathcal{T}^{p}}(X)\right\|_{F}$ for all $X \in \mathcal{M}$ since $W_{p-1} \in \mathcal{T}$ and $\mathcal{T} \subset \mathcal{T}^{p}$. This implies that

$$
\begin{aligned}
\left\|W_{p}\right\|_{F} & \leq\left\|W_{p-1}-\frac{L}{Q} \mathcal{P}_{\mathcal{T}^{p}}\left(\mathcal{A}^{p}\right)^{*} \mathcal{A}^{p} S^{p} W_{p-1}\right\|_{F} \\
& =\left\|W_{p-1}-\frac{L}{Q} \mathcal{P}_{\mathcal{T}^{p}}\left(\mathcal{A}^{p}\right)^{*} \mathcal{A}^{p} \mathcal{P}_{\mathcal{T}^{p}} S^{p} W_{p-1}\right\|_{F},
\end{aligned}
$$

where the equality is due to $S^{p} W_{p-1} \in \mathcal{T}_{p}$ and $W_{p-1} \in \mathcal{T}$. Combining this with (64), 65) and 68), leads to

$$
\begin{aligned}
& \left\|W_{p}\right\|_{F} \leq\left\|\left(I-\frac{L}{Q} \mathcal{P}_{\mathcal{T}^{p}}\left(\mathcal{A}^{p}\right)^{*} \mathcal{A}^{p}\right) S^{p} W_{p-1}\right\|_{F} \\
& +\left\|\left(I-S^{p}\right) W_{p-1}\right\|_{F} \leq \frac{1}{8}\left\|S^{p} W_{p-1}\right\|_{F}+\frac{1}{16}\left\|W_{p-1}\right\|_{F} \\
& \leq \frac{1}{4}\left\|W_{p-1}\right\|_{F} .
\end{aligned}
$$

Thus, $\forall p \in[P],\left\|W_{p}\right\|_{F} \leq(1 / 4)^{p}\left\|W_{0}\right\|_{F}=(1 / 4)^{p} \sqrt{S}$ which proves (66). As $P \geq \frac{1}{2} \log (8 \gamma \sqrt{S})$ (Def. 3), we get 67).

2) Outer Space Bound: We now turn to show that condition (36) in Lemma 5 holds. Thus, we bound the operator norm:

$$
\begin{aligned}
\left\|\mathcal{P}_{\mathcal{T}^{\perp}}\left(Y_{P}\right)\right\| & \leq \sum_{p=1}^{P}\left\|\mathcal{P}_{\mathcal{T}^{\perp}}\left(\frac{L}{Q}\left(\mathcal{A}^{p}\right)^{*} \mathcal{A}^{p} S^{p} W_{p-1}-W_{p-1}\right)\right\| \\
& \leq \sum_{p=1}^{P}\left\|\frac{L}{Q}\left(\mathcal{A}^{p}\right)^{*} \mathcal{A}^{p} S^{p} W_{p-1}-W_{p-1}\right\|
\end{aligned}
$$

where we use $W_{p-1} \in \mathcal{T}$ and the fact that the operator norm of a projection is bounded by 1 . Thus, in order to show that condition (36) holds, it remains to prove that

$$
\left\|\frac{L}{Q}\left(\mathcal{A}^{p}\right)^{*} \mathcal{A}^{p} S^{p} W_{p-1}-W_{p-1}\right\| \leq \frac{1}{4^{p+1}},
$$

for $p \in[P]$. By defining

$$
\mu_{p}=\sqrt{L} \max _{l \in \gamma_{p}}\left\|W_{p}^{*} S_{p+1} b_{l}\right\|_{2},
$$

we state the outer space bound lemma (proven in App. B-B.

Lemma 13. Let $\omega \geq 1$. Assume that $\mu_{p} \leq 4^{-p} \mu_{H}$ and $\left\|W_{p}\right\|_{F} \leq 4^{-p} \sqrt{S}$. If

$$
Q \gtrsim S\left(K \mu^{2}+N \mu_{H}^{2}\right) \log ^{2} L,
$$

then with probability $1-\mathcal{O}\left(L^{-\omega}\right)$,

$$
\left\|\frac{L}{Q}\left(\mathcal{A}^{p}\right)^{*} \mathcal{A}^{p} S^{p} W_{p-1}-W_{p-1}\right\| \leq \frac{1}{4^{p+1}}, \quad \forall p \in[P] .
$$

3) An Upper Bound for the Dual Certificate: The upper bound in (38) scales with $\|z\|_{2}$, where $z$ equals

$$
z=\frac{L}{Q} \sum_{p=1}^{P} \mathcal{A}^{p} S^{p} W_{p-1},
$$

such that $Y=\mathcal{A}^{*} z$ as in 62. We thus need to upper bound $\|z\|_{2}$ to obtain the total error bound.

Lemma 14. Let $z \in \mathcal{C}^{L}$ be given by 76 and assume $\left\|W_{p}\right\|_{F} \leq 4^{-p} \sqrt{S}$. Suppose that $\mathcal{A}^{p}$ satisfies (49) with $\delta \leq \frac{1}{4}$ on $\mathcal{T}^{p}$ for all $p \in[P]$. Then $\|z\|_{2} \lesssim P \sqrt{S}$.

Proof. By its definition 76,

$\|z\|_{2}=\frac{L}{Q} \sum_{p=1}^{P}\left\|\mathcal{A}^{p} S^{p} W_{p-1}\right\|_{F} \lesssim P \sum_{p=1}^{P}\left\|W_{p-1}\right\|_{F} \lesssim P \sqrt{S}$,

where we have used (49), 65) and $P=L / Q$ (Def. 3).

Now we can finally prove the main Theorem.

\section{E. Proof of Th. 1}

Combining the conditions on $Q$ given in Th. 7. Lemmas 13 and 19. we have that

$$
Q \gtrsim S\left(K \mu^{2} \log \left(K \mu^{2}\right)+N \mu_{H}^{2}\right) \log ^{2}(L) .
$$

Let $\Gamma_{p}$ be an admissible partition of the measurements and let $\omega>0$. Then by Def. 3 we have

$$
P \leq \log (8 \gamma \sqrt{S}) \lesssim \log L,
$$

where $\gamma \leq 2 \max \left\{1, \sqrt{\frac{N K}{L}} \mu\right\} \sqrt{\omega(\log (L+S K N))}$. As $L=$ $P Q$, we have that if

$$
L \gtrsim_{\omega} S\left(K \mu^{2} \log \left(K \mu^{2}\right)+N \mu_{H}^{2}\right) \log ^{3}(L)
$$

then we can assume that Th. 7 and Lemmas 13 and 19 hold. Thus, we can assume that conditions (23) and (49) hold with prob. $1-\mathcal{O}\left(L^{-\omega}\right)$ and constant $\delta=1 / 32$. By applying Lemma 5 with $\alpha=1 / 8 \gamma, \beta=1 / 4$ and $\delta=1 / 4$, it is enough to construct a dual certificate $Y \in \operatorname{Range} \mathcal{A}^{*}$, which 


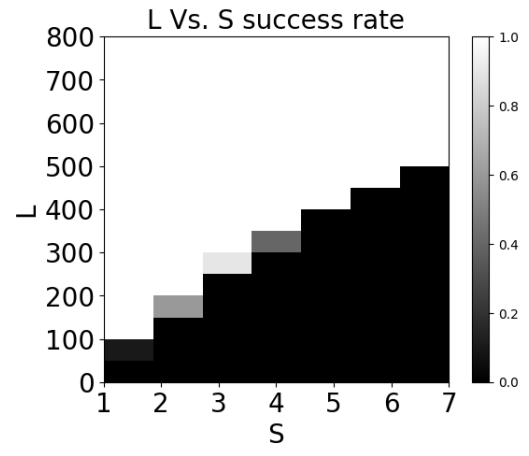

Fig. 1: Phase transition: linear empirical dependence of $\mathrm{L}$ in $\mathrm{S}$, in accordance with our theoretical results.

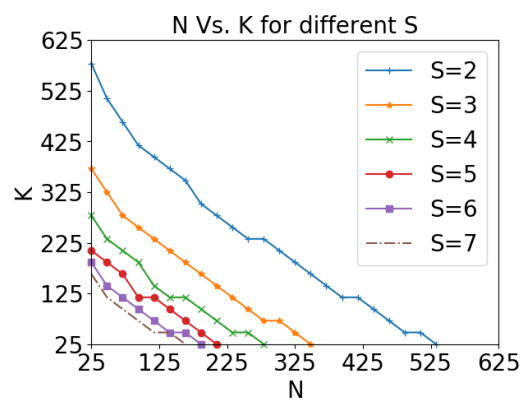

Fig. 2: Phase transition limits for a fixed $L$, varying the subspace dimensions $\mathrm{N}$ and $\mathrm{K}$. The cutoffs match the theory.

satisfies conditions (35) and 36). These conditions are met by the Golfing scheme in Lemmas 12 and 13 for a fixed $p \in[P]$. The assumptions of Lemma 12 are given by (49), thus, condition (35) applies. The assumptions of Lemma 13 are met by Lemma 19, which holds by (77) and so condition (36) holds. Thus, $Y$ defined in (62) satisfies conditions (35) and (36). Using a union bound we conclude that with probability $1-\mathcal{O}\left(L^{-\omega}\right)$ the approximate dual certificate satisfies the conditions of Lemma 5 and thus if $\hat{X}$ is the minimizer of (37) then we can bound the estimation error by 38. This error is bounded by lemmas 6 and 14 , resulting in

$$
\begin{aligned}
\left\|\hat{X}-X_{0}\right\|_{F} & \lesssim \tau(1+\gamma)\left(1+\|z\|_{2}\right) \\
& \lesssim \omega \tau \sqrt{S \max \left\{1, \frac{S K \mu^{2} N}{L}\right\} \log ^{3}(L)}
\end{aligned}
$$

\section{EXPERIMENTS}

In all the experiments, $C, M$, and $H_{r}$ are drawn from a random Gaussian distribution. $B$ consists of the $K$ first standard basis vectors, representing the blind MIMO scenario in [26] and in accordance with the results presented in [6]. The matrix reconstruction phase is done using the Matlab solver minfunc, using the heuristic solver developed by Burer and Monteiro [30] (similar to [14]). For the vector recovery, we used the Matlab non-linear equation solver fsolve with the trust-region Dogleg algorithm. All the results are measured end to end, ie. with an average error of less than $0.1 \%$ for
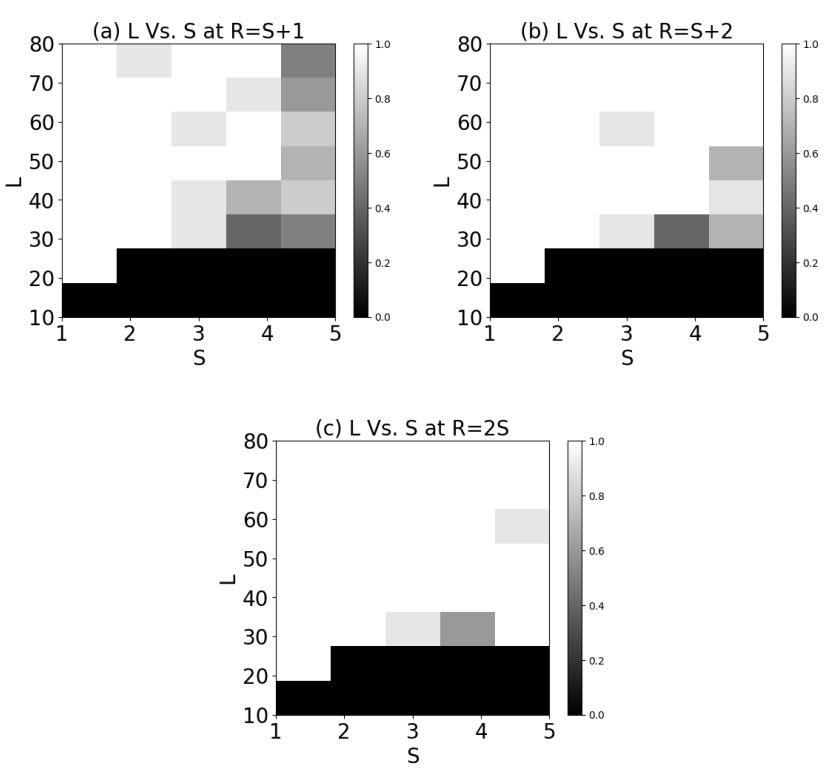

Fig. 3: Phase transition for one sided constraints. More constraints improve the basis transformation optimization.

each vector. We repeat each experiment and report the success fraction out of 10 runs.

First, we show the phase transition of the empirical reconstruction probability, changing the number of measurements at each receiver, $L$, for a different number of signals. We fix $N=30, K=25$, and report the fraction of successful reconstructions (i.e. with an average error of less than $0.1 \%$ for each vector) out of 10 experiments. The results for the two-sided constraints scenario, where $R=S$, are shown in Fig. 1] demonstrating linear dependency in accordance with the theoretical guarantees. These results are very similar to the phase transition empirical results presented in [6] but require less computational resources to achieve them.

Next, we repeat a similar experiment, only this time fixing $L=2048$ and changing $N$ and $K$. We consider the twosided constraints case, with $R=S$. Fig. 2 shows phase transition lines given the same success criterion as before, for a different number of sources. The area below the line indicates successful recovery and shows the maximal $N, K$ for a given amount of measurements per receiver. For $S=2$, $L \sim 4(N+K)$. For a larger $S=7, L \sim 11(N+K)$. The "cutoff" $N, K$ appears to be nonlinear w.r.t. $S$ and differs more for smaller values of $S$. This result is in accordance with our linear theoretical guarantees.

Fig. 3 shows the phase transition results for the one sided constraints. We use $N=6, K=5, L=10, \ldots, 80$. Fig. 3a presents the results for $R=S+1$, the minimal $R$ that allows correct recovery. The partial success areas (gray rubrics) in this chart are mostly failures to converge to some solution of the quadratic system (16) (the vector recovery stage), as opposed to wrong ambiguous solutions. A different algorithm might suggest further improved performance. Figs. $3 \mathrm{~b}(R=S+2)$ and $3 \mathrm{c}(R=2 S)$ show that adding constraints, improves the convergence and precision. The better performance of the two- 
sided setup (has fewer constraints compared to the ones sided case with $R>S+1$ ) might indicate that its structure does add up to more than the sum of its parts, and imposes some "hidden" constraints.

\section{CONCLusion}

This work presents a separable approach to blind deconvolution and demixing via convex optimization. We measure the same signals at different receivers, with different convolution kernels. Assuming all signals and kernels reside in the same subspaces, allows formulating the problem as low-rank matrices recovery. Using the assumption that the signals are normalized allows us to recover them blindly.

Our formulation allows lower complexity than [26] because we keep our matrices small. We solve a few small problems instead of a single large one. The stage we add to resolve the spanning base ambiguity of the rank-S case, is of a constant complexity of $S^{2}$, regardless of the number of receivers, and the other dimensions of the problem, i.e. $N, K, L$, which are usually much larger. Although we do not support the case of $R<S$, for the full case we have a much better complexity. We believe that our formulation can be combined with nonconvex schemes [19]-[24] to further improve their complexity. We leave this to future work.

We derive sample complexity conditions for the matrix recovery problem given in (10). This is the first work to solve this problem for rank- $S$ matrices and to supply adequate proof, which stands in line with the previous near-optimal results of the rank-1 case [17]. Future work should analyze our conjecture resolving the spanning base ambiguity. We expect the bounds to remain linear, in line with our empirical results.

\section{APPENDiX A \\ Suprema of Chaos Processes ANd Covering NUMBERS}

This section describes the necessary preliminaries used throughout the proof. We refer to [17, Sec. IV-B,C] for further reading and references. First, we define the $\gamma_{2}$ functional, a geometric quantity introduced by Talagrand [29].

Definition 15. Let $(X,|||\cdot|||)$ be a Banach space and suppose that $S \subset X$. A sequence $\left(S_{n}\right)_{n \geq 0}$ of subsets of $S$ is admissible, if $\left|S_{0}\right|=1$ and $\left|S_{n}\right|=2^{2^{n}}$ for $n \geq 1$. Then we set

$$
\gamma_{2}(S,\|\mid\| \cdot \|)=\inf _{\left(S_{n}\right)_{n \geq 0}} \sup _{s \in S} \sum_{n=0}^{\infty} 2^{n / 2} \inf _{s_{n} \in S_{n}}\left\|s-s_{n}\right\| \|,
$$

where the infimum is over all admissible sequences $\left(S_{n}\right)_{n \geq 0}$.

Furthermore, we define the distances

$$
d_{F}(\mathcal{X})=\sup _{X \in \mathcal{X}}\|X\|_{F}, d_{2 \rightarrow 2}(\mathcal{X})=\sup _{X \in \mathcal{X}}\|X\|
$$

where $\mathcal{X}$ is any set of matrices. Together with the notion of the $\gamma_{2}$ functional, we can state the following theorem that is used in the proof of Th. 7
Theorem 16 (Suprema of Chaos Processes; Th. 13 in [17]). Let $\mathcal{X}$ be a symmetric set of matrices and let $\xi$ be a random vector whose entries are $\xi_{i} \sim \mathcal{C N}(0,1)$ are independent. Set

$$
\begin{gathered}
E=\gamma_{2}(\mathcal{X},\|\cdot\|)\left(\gamma_{2}(\mathcal{X},\|\cdot\|)+d_{F}(\mathcal{X})\right) \\
V=d_{2 \rightarrow 2}(\mathcal{X})\left(\gamma_{2}(\mathcal{X},\|\cdot\|)+d_{F}(\mathcal{X})\right) \\
U=d_{2 \rightarrow 2}^{2}(\mathcal{X})
\end{gathered}
$$

Then for $t \geq 0$,

$$
\begin{array}{r}
\mathbb{P}\left(\sup _{X \in \mathcal{X}}\left|\|A \xi\|_{2}^{2}-\mathbb{E}\|A \xi\|_{2}^{2}\right| \geq c_{1} E+t\right) \leq \\
2 \exp \left(-c_{2} \min \left(\frac{t^{2}}{V^{2}}, \frac{t}{U}\right)\right)
\end{array}
$$

where the constants $c_{1}, c_{2}$ are universal.

The $\gamma_{2}$ functional can be bounded using Dudley's inequality, involving covering numbers. Recall that the covering number $N(S,\||\cdot|\|, \epsilon)$ is the minimum number of $\||\cdot|\|$-balls with radius $\epsilon$ to cover the set $S$.

Theorem 17 (Dudley's Inequality, see [29] Prop. 2.2.10, [31]). Given a set $S$ in a Banach space $(X,|| \cdot|| \mid)$, we have that

$$
\gamma_{2}(S,\||\cdot|\|) \lesssim \int_{0}^{d_{|||\cdot|||}(S)} \sqrt{\log N(S,|||\cdot| \mid, \epsilon) d \epsilon}
$$

where $d_{\||| \cdot||}(S)=\sup _{x \in S}\|x\| \|$.

We now prove Proposition (9), which is a modification to Th. 16 and used to prove Th. 7 .

Proof of Proposition 9. First we prove (51). Fix $p \in[P]$. For $Z \in \mathcal{Z}$, let $H_{Z} \in \mathbb{C}^{L \times\left|\Gamma_{p}\right| N}$ be a "block diagonal" matrix, where each block in it indexed by $l \in \Gamma_{p}$ is the row vector $\sqrt{\frac{L}{Q}} b_{l}^{*} Z \in \mathbb{C}^{1 \times N}$. Notice that

$$
\begin{aligned}
& \left\|H_{Z}\right\|_{F}^{2}=\frac{L}{Q} \sum_{l \in \Gamma_{p}}\left\|Z^{*} b_{l}\right\|_{2}^{2}=\operatorname{Tr}\left(Z Z^{*} T_{p}\right)=\left\|T_{p}^{1 / 2} Z\right\|_{F}^{2}, \\
& \left\|H_{Z}\right\|=\sqrt{\frac{L}{Q}} \max _{l \in \Gamma_{p}}\left\|b_{l}^{*} Z\right\|_{2} \leq \frac{1}{\sqrt{Q}}\|Z\|_{B} .
\end{aligned}
$$

Let $\xi^{(p)}$ be the concatenation of all $c_{l}$ where $l \in \Gamma_{p}$ and $c_{l}$ is the $l^{\text {th }}$ column of $C^{T}$. Then

$$
\begin{aligned}
\frac{L}{Q}\left\|\mathcal{A}^{p}(Z)\right\|_{2}^{2}= & \frac{L}{Q} \sum_{l \in \Gamma_{p}}\left|\mathcal{A}^{p}(Z)(l)\right|^{2}= \\
& \frac{L}{Q} \sum_{l \in \Gamma_{p}}\left|b_{l}^{*} Z c_{l}\right|^{2}=\left\|H_{Z} \xi^{(p)}\right\|_{2}^{2} .
\end{aligned}
$$

Notice that

$$
\mathbb{E}\left[\left\|H_{Z} \xi^{(p)}\right\|_{2}^{2}\right]=\left\|T_{p}^{1 / 2} Z\right\|_{F}^{2}=\left\|H_{Z}\right\|_{F}^{2},
$$

and thus

$$
\begin{aligned}
& \sup _{Z \in \mathcal{Z}}\left|\left\|H_{Z} \xi^{(p)}\right\|_{2}^{2}-\mathbb{E}\left[\left\|H_{Z} \xi^{(p)}\right\|_{2}^{2}\right]\right|= \\
& \sup _{Z \in \mathcal{Z}}\left|\frac{L}{Q}\left\|\mathcal{A}^{p}(Z)\right\|_{2}^{2}-\left\|T_{P}^{1 / 2} Z\right\|_{F}^{2}\right| .
\end{aligned}
$$


To get (51) we just need to apply Th. 16. The proof for 52 is similar, where the "diagonal" elements in $H_{Z}$ are non zero for $l \in[L]$, and $T_{p}$ is replaced with $\sum_{l \in[L]} b_{l} b_{l}^{*}=I$.

\section{APPENDIX B}

\section{PROOF OF DifFERENT LEMMAS}

\section{A. Proof of Lemma 6}

Proof of Lemma 6 Recall $\mathcal{A}(Z)=\left[\left\langle A_{1}, Z\right\rangle, \ldots,\left\langle A_{L}, Z\right\rangle\right]^{T}$ where $A_{l}=\sqrt{L} B^{*} F^{*} \operatorname{diag}\left(F_{l}\right) \bar{F} \bar{C}=\hat{b}_{l} \hat{c}_{l}^{*}$. In order to bound $\gamma$, we will estimate the norms of the expected operator norms of $\mathcal{A}^{*} \mathcal{A}$ and $\mathcal{A A}^{*}$. Starting with the former,

$$
\begin{gathered}
\mathbb{E}\left[\mathcal{A}^{*} \mathcal{A}(Z)\right]=\sum_{l \in[L]} \mathbb{E}\left[\mathcal{A}(Z)(l) \hat{b}_{l} \hat{c}_{l}^{*}\right]= \\
\sum_{l \in[L]} \mathbb{E}\left[\hat{b}_{l} \hat{b}_{l}^{*} Z \hat{c}_{l} \hat{c}_{l}^{*}\right]=\sum_{l \in[L]} \hat{b}_{l} \hat{b}_{l}^{*} Z=Z,
\end{gathered}
$$

meaning that $\mathbb{E}\left[\mathcal{A}^{*} \mathcal{A}\right]=\mathbb{I}$. Moving to the latter,

$$
\begin{aligned}
& \mathbb{E}\left[\mathcal{A} \mathcal{A}^{*} y(l)\right]=\mathbb{E}\left[\hat{b}_{l}^{*} \mathcal{A}^{*}(y) \hat{c}_{l}\right]=\sum_{l^{*} \in[L]} \mathbb{E}\left[\hat{b}_{l}^{*} \hat{b}_{l^{\prime}} y\left(l^{\prime}\right) \hat{c}_{l^{*}}^{*} \hat{c}_{l}\right] \\
& =y(l) \sum_{l^{*} \in[L]} \mathbb{E}\left[\hat{b}_{l}^{*} \hat{b}_{l^{\prime}} \hat{c}_{l^{\prime}}^{*} \hat{c}_{l}\right]=y(l) N\left\|\hat{b}_{l}\right\|_{2}^{2} .
\end{aligned}
$$

Thus, $\mathbb{E}\left[\mathcal{A A}^{*}\right]=\operatorname{diag}\left(N\left\|\hat{b_{1}}\right\|_{2}^{2}, \ldots, N\left\|\hat{b_{L}}\right\|_{2}^{2}\right)$. Combined with the definition of $\mu$ in [24], we get $\left\|\mathbb{E}\left[\mathcal{A A}^{*}\right]\right\| \leq \frac{N K \mu^{2}}{L}$. Thus, $\sigma^{2}=\max \left\{\left\|\mathbb{E}\left[\mathcal{A}^{*} \mathcal{A}\right]\right\|,\left\|\mathbb{E}\left[\mathcal{A} \mathcal{A}^{*}\right]\right\|\right\} \leq \max \left\{1, \frac{N K \mu^{2}}{L}\right\}$.

Applying Corollary 10 in [17] with $t=\omega \log (L)$,

$\|\mathcal{A}\| \leq \max \left\{1, \sqrt{\frac{N K}{L}} \mu\right\} \sqrt{(2 \omega \log L+\log (L+S K N))}$ with probability exceeding $1-2 L^{-\omega}$.

\section{B. Proof of Lemma 13}

We will use Th. 9 in [17] to bound [72].

Lemma 18 (Matrix Bernstein Inequality, Th. 9 in [17]). Let $\alpha \in[1, \infty)$ and let $X_{1}, \ldots, X_{n} \in \mathbb{C}^{d_{1} \times d_{2}}$ be independent random matrices that satisfy $\mathbb{E}\left[X_{i}\right]=0$ for all $i \in[n]$. Set $R_{\psi_{\alpha}}=\max _{i \in[n]}\|\| X_{i}\|\|_{\psi_{\alpha}}$ and $\sigma^{2}=$ $\max \left\{\left\|\sum_{i=1}^{n} \mathbb{E}\left[X_{i} X_{i}^{*}\right]\right\|,\left\|\sum_{i=1}^{n} \mathbb{E}\left[X_{i}^{*} X_{i}\right]\right\|\right\}$. Set $Z=$ $\sum_{i=1}^{n} X_{i}$. Then with probability at least $1-e^{-t}$,

$$
\begin{aligned}
& \|Z\| \lesssim \max \left\{\sigma \sqrt{t+\log \left(d_{1}+d_{2}\right)}\right. \\
& \left.R_{\psi_{\alpha}}\left(\log \left(1+\frac{n R_{\psi_{\alpha}}^{2}}{\sigma^{2}}\right)\right)^{1 / \alpha}\left(t+\log \left(d_{1}+d_{2}\right)\right)\right\} .
\end{aligned}
$$

Proof of Lemma 13. First, notice that for all $l \in \Gamma_{p}$,

$$
\begin{aligned}
\left(\mathcal{A}^{p} S^{p} W_{p-1}\right)(l) & =b_{l}^{*} S^{p} W_{p-1} c_{l}, \\
\left(\left(\mathcal{A}^{p}\right)^{*} \mathcal{A}^{p} S^{p} W_{p-1}\right) & =\sum_{l \in \Gamma_{p}} b_{l} b_{l}^{*} S^{p} W_{p-1} c_{l} c_{l}^{*} .
\end{aligned}
$$

Since by its definition, $S_{p}=\left(T_{p}\right)^{-1}$ (see (27)), we have that

$$
W_{p-1}=T_{p} S_{p} W_{p-1}=\frac{L}{Q} b_{l} b_{l}^{*} S_{p} W_{p-1} .
$$

For simplicity of notation, define

$$
w_{l}=W_{p-1}^{*} S_{p} b_{l} .
$$

Thus, we can write

$$
\begin{aligned}
& \frac{L}{Q}\left(\mathcal{A}^{p}\right)^{*} \mathcal{A}^{p} S^{p} W_{p-1}-W_{p-1}=\frac{L}{Q} \sum_{l \in \Gamma_{p}} b_{l} w_{l}^{*} c_{l} c_{l}^{*}- \\
& \frac{L}{Q} \sum_{l \in \Gamma_{p}} b_{l} w_{l}^{*}=\frac{L}{Q} \sum_{l \in \Gamma_{p}} b_{l} w_{l}^{*}\left(c_{l} c_{l}^{*}-I\right)=\sum_{l \in \Gamma_{p}} Z_{l},
\end{aligned}
$$

where

$$
Z_{l} \triangleq \frac{L}{Q} b_{l} w_{l}^{*}\left(c_{l} c_{l}^{*}-I\right) .
$$

We now asses the the relevant components to use Lemma 18 We start with the expectation values of $Z_{l} Z_{l}^{*}$ and $Z_{l}^{*} Z_{l}$.

$$
\begin{aligned}
& \mathbb{E}\left[Z_{l} Z_{l}^{*}\right]=\mathbb{E}\left[\frac{L^{2}}{Q^{2}} b_{l} w_{l}^{*}\left(c_{l} c_{l}^{*}-I\right)^{2} w_{l} b_{l}^{*}\right] \\
& =\frac{L^{2}}{Q^{2}} b_{l} w_{l}^{*} \mathbb{E}\left[\left(c_{l} c_{l}^{*}-I\right)^{2}\right] w_{l} b_{l}^{*}=\frac{L^{2}}{Q^{2}} N\left\|w_{l}\right\|_{2}^{2} b_{l} b_{l}^{*},
\end{aligned}
$$

since $\mathbb{E}\left[\left(c_{l} c_{l}^{*}-I\right)^{2}\right]=N I$ by Lemma 11 in [14].

$\mathbb{E}\left[Z_{l}^{*} Z_{l}\right]=\mathbb{E}\left[\frac{L^{2}}{Q^{2}}\left(c_{l} c_{l}^{*}-I\right) w_{l} b_{l}^{*} b_{l} w_{l}^{*}\left(c_{l} c_{l}^{*}-I\right)\right]=$

$\frac{L^{2}}{Q^{2}}\left\|b_{l}\right\|_{2}^{2} \mathbb{E}\left[\left(c_{l} c_{l}^{*}-I\right) w_{l} w_{l}^{*}\left(c_{l} c_{l}^{*}-I\right)\right]=\frac{L^{2}}{Q^{2}}\left\|b_{l}\right\|_{2}^{2}\left\|w_{l}\right\|_{2}^{2} I$,

since $\mathbb{E}\left[\left(c_{l} c_{l}^{*}-I\right) w_{l} w_{l}^{*}\left(c_{l} c_{l}^{*}-I\right)\right]=\left\|w_{l}\right\|_{2}^{2} I$ by Lemma 12 in [14]. Furthermore, we have that

$$
\begin{aligned}
\left\|\sum_{l \in \Gamma_{p}} \mathbb{E}\left[Z_{l} Z_{l}^{*}\right]\right\| & \leq \frac{L^{2} N}{Q^{2}} \max _{l \in \Gamma_{p}}\left(\left\|w_{l}\right\|_{2}^{2}\right)\left\|\sum_{l \in \Gamma_{p}} b_{l} b_{l}^{*}\right\| \\
& \leq \frac{N}{Q} \mu_{p-1}^{2}\left\|T_{p}\right\| \lesssim \frac{16^{-p} N \mu_{H}^{2}}{Q}
\end{aligned}
$$

due to the lemma's assumptions and 28.

$$
\begin{aligned}
& \left\|\sum_{l \in \Gamma_{p}} \mathbb{E}\left[Z_{l}^{*} Z_{l}\right]\right\| \leq \frac{L^{2}}{Q^{2}} \max _{l \in \Gamma_{p}}\left(\left\|b_{l}\right\|_{2}^{2}\right)\left\|\sum_{l \in \Gamma_{p}}\right\| w_{l}\left\|_{2}^{2}\right\| \\
& \leq \frac{L K \mu^{2}}{Q^{2}} \sum_{l \in \Gamma_{p}} \operatorname{Tr}\left(W_{p-1}^{*} S_{p} b_{l} b_{l}^{*} S_{p} W_{p-1}\right) \\
& =\frac{K \mu^{2}}{Q}\left\|S_{p}^{1 / 2} W_{p-1}\right\|_{F}^{2} \lesssim 16^{-p} \frac{S K \mu^{2}}{Q} .
\end{aligned}
$$

Thus, we have

$$
\begin{aligned}
\sigma^{2} & =\max \left\{\left\|\sum_{l \in \Gamma_{p}} \mathbb{E}\left[Z_{l} Z_{l}^{*}\right]\right\|,\left\|\sum_{l \in \Gamma_{p}} \mathbb{E}\left[Z_{l}^{*} Z_{l}\right]\right\|\right\} \quad(101) \\
& \lesssim \frac{16^{-p}}{Q} \max \left(S K \mu^{2}, N \mu_{H}^{2}\right) \leq \frac{16^{-p}}{Q}\left(S K \mu^{2}+N \mu_{H}^{2}\right) .
\end{aligned}
$$


Now we estimate $R_{\psi_{\alpha}}=\max \|\| Z_{l}\|\|_{\psi_{\alpha}}$, where $\|\cdot\|_{\psi_{\alpha}}$ is the Orlicz norm (Def. 7 in [17]).

\|\|$Z_{l}\|\|_{\psi_{1}}=\|\| \frac{L}{Q} b_{l} w_{l}^{*}\left(c_{l} c_{l}^{*}-I\right)\|\|_{\psi_{1}}$

$\leq \frac{L}{Q}\|\| b_{l} w_{l}^{*}\left(c_{l} c_{l}^{*}-I\right)\left\|_{2}\right\|_{\psi_{1}} \leq \frac{L}{Q}\left\|b_{l}\right\|_{2}\|\| w_{l}^{*}\left(c_{l} c_{l}^{*}-I\right)\left\|_{2}\right\|_{\psi_{1}}$

$\lesssim \frac{L \sqrt{N}}{Q}\left\|b_{l}\right\|_{2}\left\|w_{l}\right\|_{2} \lesssim \frac{L \sqrt{N}}{Q} \sqrt{\frac{K}{L}} \mu \frac{\mu_{p-1}}{\sqrt{L}}$

$\lesssim 4^{-p} \frac{\sqrt{N K} \mu \mu_{H}}{Q} \lesssim 4^{-p} \frac{\left(K \mu^{2}+N \mu_{H}^{2}\right)}{Q}$,

where the second inequality is due to Lemma 39 in [17], and the last step follows from this lemma's assumptions. We continue to asses the size $\frac{\left|\Gamma_{p}\right| R_{\psi_{\alpha}}^{2}}{\sigma^{2}}$. We have that

$$
\begin{aligned}
\frac{\left|\Gamma_{p}\right| R_{\psi_{\alpha}}^{2}}{\sigma^{2}} & \lesssim \frac{Q 4^{-2 p \frac{N K \mu^{2} \mu_{H}^{2}}{Q^{2}}}}{\frac{16^{-p}}{Q} \max \left(S K \mu^{2}, N \mu_{H}^{2}\right)} \\
& \lesssim \frac{K \mu^{2} N \mu_{H}^{2}}{N \mu_{H}^{2}}=K \mu^{2} \leq L .
\end{aligned}
$$

Finally, we can set these sizes in Lemma 18 with $t=(\omega+$ 1) $\log L, \alpha=1$ and get with probability $1-\mathcal{O}\left(L^{-\omega-1}\right)$,

$$
\left\|\sum_{l \in \Gamma_{p}} Z_{l}\right\| \lesssim_{\omega} 4^{-p} \max \left\{\begin{array}{l}
\sqrt{\frac{\left(S K \mu^{2}+N \mu_{H}^{2}\right) \log L}{Q}}, \\
\left.\frac{K \mu^{2}+N \mu_{H}^{2}}{Q} \log ^{2} L\right\},
\end{array}\right.
$$

for a $p \in[P]$. Taking the union bound for all $p \in[P]$, we get $\left\|\frac{L}{Q}\left(\mathcal{A}^{p}\right)^{*} \mathcal{A}^{p} S^{p} W_{p-1}-W_{p-1}\right\| \leq \frac{1}{4^{p+1}}$ with prob. of at least $1-P \mathcal{O}\left(L^{-\omega-1}\right)=1-\mathcal{O}\left(L^{-\omega}\right)$, which finishes the proof.

It thus remains to prove that $\mu_{p} \leq \frac{1}{4} \mu_{p-1}$.

Lemma 19. Let $\omega \geq 1$. if

$$
Q \gtrsim S \max \left(K \mu^{2}, N \mu_{H}^{2}\right) \log ^{2} L
$$

then with probability $1-\mathcal{O}\left(L^{-\omega}\right)$ it holds that $\mu_{p} \leq \frac{1}{4} \mu_{p-1}$ for all $p \in[P-1]$.

Proof. Recall that by the definition of $\mu_{p}$ in (73), we need to show that for all $l \in \Gamma_{p}$ and for all $p \in[P-1]$,

$$
\sqrt{L}\left\|W_{p}^{*} S_{p+1} b_{l}\right\|_{2} \leq \frac{1}{4} \mu_{p-1}
$$

By [69), we have that $W_{p}=W_{p-1}-\frac{L}{Q} \mathcal{P}_{\mathcal{T}}\left(\mathcal{A}^{p}\right)^{*} \mathcal{A}^{p} S^{p} W_{p-1}$. Thus, we can use (31), (33) together with (95) to write

$$
\begin{aligned}
& W_{p}=\frac{L}{Q} \sum_{j \in \Gamma_{p}}\left(\quad \hat{H} \hat{H}^{*} b_{j} w_{j}^{*}\left(I-c_{j} c_{j}^{*}\right)+\right. \\
& \left.\left(I-\hat{H} \hat{H}^{*}\right) b_{j} w_{j}^{*}\left(I-c_{j} c_{j}^{*}\right) \hat{M} \hat{M}^{*}\right),
\end{aligned}
$$

where $w_{j}=W_{p-1}^{*} S_{p} b_{j}$ as in (94) and $W_{p-1} \in \mathcal{T}$. Using the triangle inequality, we have that

$$
\begin{gathered}
\left\|W_{p}^{*} S_{p+1} b_{l}\right\|_{2} \leq\left\|\frac{L}{Q} \sum_{j \in \Gamma_{p}}\left(I-c_{j} c_{j}^{*}\right) w_{j} b_{j}^{*} \hat{H} \hat{H}^{*} S_{p+1} b_{l}\right\|_{2}+ \\
\left\|\frac{L}{Q} \sum_{j \in \Gamma_{p}} \hat{M} \hat{M}^{*}\left(I-c_{j} c_{j}^{*}\right) w_{j} b_{j}^{*}\left(I-\hat{H} \hat{H}^{*}\right) S_{p+1} b_{l}\right\|_{2} \\
\triangleq\left\|\sum_{j \in \Gamma_{p}} u_{j}\right\|_{2}+\left\|\sum_{j \in \Gamma_{p}} v_{j}\right\|_{2}
\end{gathered}
$$

We now use Lemma 18 again to bound the two summands. For the first part, we have

$$
\begin{aligned}
\left\|\sum_{j \in \Gamma_{p}} E\left[u_{j} u_{j}^{*}\right]\right\| & =\frac{L^{2}}{Q^{2}}\left\|\sum_{j \in \Gamma_{p}} w_{j} b_{j}^{*} \hat{H} \hat{H}^{*} S_{p+1} b_{l}\right\|_{2}^{2} \quad \text { (109) } \\
& \leq \frac{L^{2}}{Q^{2}} \sum_{j \in \Gamma_{p}, s \in[S]}\left\|w_{j} b_{j}^{*} \hat{h}_{s} \hat{h}_{s}^{*} S_{p+1} b_{l}\right\|_{2}^{2} \\
& =\frac{L^{2}}{Q^{2}} \sum_{s \in[S]}\left|\hat{h}_{s}^{*} S_{p+1} b_{l}\right|^{2} \sum_{j \in \Gamma_{p}}\left|b_{j}^{*} \hat{h}_{s}\right|^{2}\left\|w_{j}\right\|_{2}^{2} \\
& \leq \frac{\mu_{H}^{2} \mu_{p-1}^{2}}{Q L} \sum_{s \in[S]}\left\|T_{p}^{1 / 2} \hat{h}_{s}\right\|_{2}^{2} \lesssim \frac{S \mu_{H}^{2} \mu_{p-1}^{2}}{Q L},
\end{aligned}
$$

where we have used Lemma 12 in [14] again and by (63) and the definitions in 29), (73), 26) and 33). Similarly,

$$
\begin{aligned}
\left\|\sum_{j \in \Gamma_{p}} E\left[u_{j}^{*} u_{j}\right]\right\| & \leq \frac{L^{2} N}{Q^{2}} \sum_{j \in \Gamma_{p}, s \in[S]}\left\|w_{j} b_{j}^{*} \hat{h}_{s} \hat{h}_{s}^{*} S_{p+1} b_{l}\right\|_{2}^{2} \\
& \lesssim \frac{S N \mu_{H}^{2} \mu_{p-1}^{2}}{Q L}
\end{aligned}
$$

again using Lemma 11 in $[14]$, leading to $\sigma^{2} \lesssim \frac{S N \mu_{H}^{2} \mu_{p-1}^{2}}{Q L}$. Furthermore,

$$
\begin{aligned}
R_{\psi_{1}} & =\max _{j \in \Gamma_{p}}\|\| \frac{L}{Q}\left(I-c_{j} c_{j}^{*}\right) w_{j} b_{j}^{*} \hat{H} \hat{H}^{*} S_{p+1} b_{l}\left\|_{2}\right\|_{\psi_{1}}(111) \\
& =\frac{L}{Q} \max _{j \in \Gamma_{p}}\|\| \sum_{s \in[S]}\left(I-c_{j} c_{j}^{*}\right) w_{j} b_{j}^{*} \hat{h}_{s} \hat{h}_{s}^{*} S_{p+1} b_{l}\left\|_{2}\right\|_{\psi_{1}} \\
& \leq \frac{L}{Q} \frac{\mu_{H}^{2}}{L} S \max _{j \in \Gamma_{p}}\|\|\left(I-c_{j} c_{j}^{*}\right) w_{j}\left\|_{2}\right\|_{\psi_{1}} \\
& \lesssim \frac{L}{Q} \frac{\mu_{H}^{2}}{L} S \max _{j \in \Gamma_{p}} \sqrt{N}\left\|w_{j}\right\|_{2} \leq \frac{S \sqrt{N} \mu_{H}^{2} \mu_{p-1}}{Q \sqrt{L}} .
\end{aligned}
$$

Now, we can assess

$$
\frac{\left|\Gamma_{p}\right| R_{\psi_{1}}^{2}}{\sigma^{2}} \lesssim Q \frac{S^{2} N \mu_{H}^{4} \mu_{p-1}^{2}}{Q^{2} L} \frac{Q L}{S N \mu_{H}^{2} \mu_{p-1}^{2}}=S \mu_{H}^{2} \lesssim S L .
$$

Finally, we can write with $t=(\omega+1) \log L, \alpha=1$

$$
\begin{aligned}
& \left\|\frac{L}{Q} \sum_{j \in \Gamma_{p}}\left(I-c_{j} c_{j}^{*}\right) w_{j} b_{j}^{*} \hat{H} \hat{H}^{*} S_{p+1} b_{l}\right\|_{2} \lesssim \\
& \frac{\mu_{p-1}}{\sqrt{L}} \max \left\{\sqrt{\frac{S N \mu_{H}^{2}}{Q} \log L}, \frac{S \sqrt{N} \mu_{H}^{2}}{Q} \log ^{2} L\right\}
\end{aligned}
$$


with a probability of at least $1-\mathcal{O}\left(L^{-\omega-1}\right)$ for a fixed $p \in$ $[P]$. Taking the union bound for all $p \in[P]$, we get that if $Q \gtrsim S N \mu_{H}^{2} \log ^{2} L$, then the first summand in $(108)$ is bounded as required by $(106)$ with probability of at least $1-$ $P \mathcal{O}\left(L^{-\omega-1}\right)=1-\mathcal{O}\left(L^{-\omega}\right)$.

We now repeat the same process for the second summand in (108). We use the $Z_{j}$ notation defined in (96).

$$
\begin{aligned}
& \left\|\sum_{j \in \Gamma_{p}} E\left[v_{j} v_{j}^{*}\right]\right\| \leq \sum_{j \in \Gamma_{p}} E \| \hat{M} \hat{M}^{*} Z_{j}^{*}\left(I-\hat{H} \hat{H}^{*}\right) S_{p+1} b_{l} . \\
& \leq \sum_{j \in \Gamma_{p}} E\left\|Z_{j}^{*}\left(I-\hat{H} \hat{H}^{*}\right) S_{p+1} b_{l} b_{l}^{*} S_{p+1}\left(I-\hat{H} \hat{H}^{*}\right) Z_{j}\right\| \\
& =\frac{L^{2}}{Q^{2}} \sum_{j \in \Gamma_{p}} E \|\left(I-c_{j} c_{j}^{*}\right) w_{j} b_{j}^{*}\left(I-\hat{H} \hat{H}^{*}\right) S_{p+1} b_{l} \cdot \\
& =\frac{L^{2}}{Q^{2}} \sum_{j \in \Gamma_{p}}\left\|w_{j} b_{j}^{*}\left(I-\hat{H} \hat{H}^{*}\right) S_{p+1} b_{l}\right\|_{2}^{2} \\
& \leq \frac{L^{2}}{Q^{2}} \sum_{j \in \Gamma_{p}}\left\|w_{j}\right\|_{2}^{2}\left|b_{j}^{*}\left(I-\hat{H} \hat{H}^{*}\right) S_{p+1} b_{l}\right|^{2} \\
& \leq \frac{L^{2}}{Q^{2}} \frac{\mu_{p-1}^{2}}{L} \frac{Q}{L}\left\|T_{p}^{1 / 2}\left(I-\hat{H} \hat{H}^{*}\right) S_{p+1} b_{l}\right\|^{2} \\
& \leq \frac{L^{2}}{Q^{2}} \frac{\mu_{p-1}^{2}}{L} \frac{Q}{L}\left\|T_{p}^{1 / 2}\right\|^{2}\left\|\left(I-\hat{H} \hat{H}^{*}\right)\right\|^{2}\left\|S_{p+1}\right\|^{2}\left\|b_{l}\right\|^{2} \\
& \lesssim \frac{L^{2}}{Q^{2}} \frac{\mu_{p-1}^{2}}{L} \frac{Q}{L}\left\|b_{l}\right\|^{2} \leq \frac{L^{2}}{Q^{2}} \frac{\mu_{p-1}^{2}}{L} \frac{Q}{L} \frac{K \mu^{2}}{L}=\frac{K \mu^{2} \mu_{p-1}^{2}}{Q L}
\end{aligned}
$$

where we have used Lemma 12 in [14] again and by [63 and the definitions in 29, (73), 26. and (33).

Similarly,

$$
\begin{aligned}
& \left\|\sum_{j \in \Gamma_{p}} E\left[v_{j}^{*} v_{j}\right]\right\| \\
& \leq \frac{L^{2}}{Q^{2}} \sum_{j \in \Gamma_{p}} E \| b_{l}^{*} S_{p+1}\left(I-\hat{H} \hat{H}^{*}\right) b_{j} w_{j}^{*}\left(I-c_{j} c_{j}^{*}\right) \hat{M} \hat{M}^{*} . \\
& =\frac{L^{2} S}{Q^{2}} \sum_{j \in \Gamma_{p}}\left\|w_{j} b_{j}^{*}\left(I-\hat{H} \hat{H}^{*}\right) S_{p+1} b_{l}\right\|_{2}^{2} \lesssim \frac{S K \mu_{j}^{2} \mu_{p-1}^{2}}{Q L} w_{j}^{*}\left(I-\hat{H} \hat{H}^{*}\right) S_{p+1} b_{l}
\end{aligned}
$$

again using Lemma 12 in $[14]$. Thus, $\sigma^{2} \lesssim \frac{S K \mu^{2} \mu_{p-1}^{2}}{Q L}$. Furthermore,

$$
\begin{aligned}
& R_{\psi_{1}}=\max _{j \in \Gamma_{p}}\|\| \frac{L}{Q} \hat{M} \hat{M}^{*}\left(I-c_{j} c_{j}^{*}\right) w_{j} b_{j}^{*}\left(I-\hat{H} \hat{H}^{*}\right) S_{p+1} b_{l}\left\|_{2}\right\|_{\psi} \\
& =\max _{j \in \Gamma_{p}}\|\| \frac{L}{Q} \hat{M}^{*}\left(I-c_{j} c_{j}^{*}\right) w_{j} b_{j}^{*}\left(I-\hat{H} \hat{H}^{*}\right) S_{p+1} b_{l}\left\|_{2}\right\|_{\psi_{1}} \\
& \leq \frac{L}{Q} \max _{j \in \Gamma_{p}}\left|b_{j}^{*}\left(I-\hat{H} \hat{H}^{*}\right) S_{p+1} b_{l}\right| \sum_{s \in[S]}\left\|\hat{m}_{s}^{*}\left(I-c_{j} c_{j}^{*}\right) w_{j}\right\|_{\psi_{1}} \\
& \leq \frac{L}{Q} \frac{K \mu^{2}}{L} \sum_{s \in[S]}\left\|\hat{m}_{s}^{*}\right\|_{2} \max _{j \in \Gamma_{p}}\left\|w_{j}\right\|_{2} \leq \frac{S K \mu^{2} \mu_{p-1}}{Q \sqrt{L}}
\end{aligned}
$$

where we have used Lemma 39 in [17]. Now, we can assess

$$
\frac{\left|\Gamma_{p}\right| R_{\psi_{1}}^{2}}{\sigma^{2}} \lesssim Q \frac{S^{2} K^{2} \mu^{4} \mu_{p-1}^{2}}{Q^{2} L} \frac{Q L}{S K \mu^{2} \mu_{p-1}^{2}}=S K \mu^{2} \leq S L .(117)
$$

Finally, we can write with $t=(\omega+1) \log L, \alpha=1$

$$
\begin{gathered}
\left\|\frac{L}{Q} \sum_{j \in \Gamma_{p}} \hat{M} \hat{M}^{*}\left(I-c_{j} c_{j}^{*}\right) w_{j} b_{j}^{*}\left(I-\hat{H} \hat{H}^{*}\right) S_{p+1} b_{l}\right\|_{2} \\
\lesssim \frac{\mu_{p-1}}{\sqrt{L}} \max \left\{\sqrt{\frac{S K \mu^{2} \log L}{Q}}, \frac{S K \mu^{2}}{Q} \log ^{2} L\right\}
\end{gathered}
$$

with a probability of at least $1-\mathcal{O}\left(L^{-\omega-1}\right)$ for a fixed $p \in$ $[P]$. Taking the union bound for all $p \in[P]$, we get that if $Q \gtrsim S K \mu^{2} \log ^{2} L$, then the second summand in (108) is bounded as required by $(106)$ with probability of at least $1-$ $P \mathcal{O}\left(L^{-\omega-1}\right)=1-\mathcal{O}\left(L^{-\omega}\right)$, which finishes the proof.

\section{Proof of Lemma 10}

Proof of Lemma 10. To prove this lemma, we use the following two technical lemmas. The proof of the first appears in Appendix B-D and of the second in [17]. First, let us denote the unit ball with respect to $\|\cdot\|_{2}$ by $\bar{B}(0,1)$ (throughout the paper).

Lemma 20. Let $\mathcal{B}^{M}$ be defined by 53. Then

$$
\begin{array}{r}
N\left(\mathcal{B}^{M},\|\cdot\|_{B}, \epsilon\right) \leq N\left(B(0,1) \subset \mathbb{R}^{S},\|\cdot\|_{2}, \frac{\epsilon}{2 \sqrt{K \mu}}\right) \\
\cdot N^{S}\left(B(0,1) \subset \mathbb{C}^{K},\|\cdot\|_{B}, \frac{\epsilon}{2}\right)
\end{array}
$$

Lemma 21. (A private case of Lemma 27 in [17]).

$$
\log N\left(B(0,1) \subset \mathbb{C}^{K},\|\cdot\|_{B}, \frac{\epsilon}{2}\right) \lesssim \frac{K \mu^{2}}{\epsilon^{2}} \log L .
$$

We now turn to prove Lemma 10 The first inequality 56 follows the fact that

$$
d_{F}(\mathcal{X})=\sup _{X \in \mathcal{X}}\|X\|_{F} \leq \sup _{X \in \mathcal{W}^{p}}\|X\|_{F} \leq 3
$$

where the last inequality holds since $\mathcal{W}^{p}=\mathcal{B}^{M}+\mathcal{B}^{H}+\mathcal{B}^{S^{p} H}$ and all the elements in these sets are normalized.

To prove the second inequality (57), we use the definitions of $d_{B}(\mathcal{X})$ and $\|\cdot\|_{B}$, to get

$$
\begin{gathered}
d_{B}(\mathcal{X})=\sup _{X \in \mathcal{X}}\|X\|_{B}=\sup _{X \in \mathcal{X}} \sqrt{L} \max _{l \in[L]}\left\|X^{*} b_{l}\right\|_{F} \leq \text { (122) } \\
\sup _{X \in \mathcal{X}} \sqrt{L}\|X\|_{F} \max _{l \in[L]}\left\|b_{l}\right\|_{2} \leq \sup _{X \in \mathcal{X}}\|X\|_{F} \sqrt{K} \mu \leq 3 \sqrt{K} \mu,
\end{gathered}
$$

where the first inequality is due to the Frobenius norm properties, the second is due to the definition of $\mu$ in 24 and the last is due to 56 .

For [58, we can use Lemma 12 in [17] to obtain

$$
\begin{aligned}
& \gamma_{2}\left(\mathcal{W}^{p},\|\cdot\|_{B}\right) \lesssim \\
& \gamma_{2}\left(\mathcal{B}^{H},\|\cdot\|_{B}\right)+\gamma_{2}\left(\mathcal{B}^{M},\|\cdot\|_{B}\right)+\gamma_{2}\left(\mathcal{B}^{S^{p} H},\|\cdot\|_{B}\right)
\end{aligned}
$$

where $\gamma_{2}\left(\mathcal{W},\|\cdot\|_{B}\right)$ is bounded analogously. 
First we bound $\gamma_{2}\left(\mathcal{B}^{H},\|\cdot\|_{B}\right)$. Let $\mathbf{U}=\hat{H} U^{*}, \mathbf{V}=\hat{H} V^{*} \in$ $\mathcal{B}^{M}$. Then

$$
\begin{aligned}
& \|\mathbf{U}-\mathbf{V}\|_{B}^{2}=\left\|\hat{H}\left(U^{*}-V^{*}\right)\right\|_{B}^{2}= \\
& =\operatorname{Lmax}_{l \in[L]}\left\|(U-V) \hat{H}^{*} b_{l}\right\|_{2}^{2} \leq L \max _{l \in[L]} \sum_{s \in[S]}\left\|\hat{h}_{s}^{*} b_{l}\left(u_{s}-v_{s}\right)\right\|_{2}^{2} \\
& =\operatorname{Lmax}_{l \in[L]} \sum_{s \in[S]}\left|\hat{h}_{s}^{*} b_{l}\right|^{2}\left\|\left(u_{s}-v_{s}\right)\right\|_{2}^{2} \leq \mu_{H}^{2} \sum_{s \in[S]}\left\|\left(u_{s}-v_{s}\right)\right\|_{2}^{2} \\
& =\mu_{H}^{2}\|(U-V)\|_{F}^{2}=\mu_{H}^{2}\|\mathbf{U}-\mathbf{V}\|_{F}^{2}
\end{aligned}
$$

where the inequality is due to 29 and the last equality holds because the Frobenius norm is unitary invariant. Using (124) followed by Dudley's inequality (Th. 17), implies

$$
\begin{aligned}
& \gamma_{2}\left(\mathcal{B}^{H},\|\cdot\|_{B}\right) \leq \mu_{H} \gamma_{2}\left(\mathcal{B}^{H},\|\cdot\|_{F}\right) \lesssim \\
& \mu_{H} \int_{0}^{1} \sqrt{\log N\left(\mathcal{B}^{H},\|\cdot\|_{F}, \epsilon\right)} d \epsilon \lesssim \mu_{H} \sqrt{S N},
\end{aligned}
$$

where the last inequality holds since $\left(\mathcal{B}^{H},\|\cdot\|_{F}\right)$ is isometric to $\left(B(0,1) \subset \mathbb{R}^{2 S N},\|\cdot\|_{2}\right)$ and a standard volumetric estimate.

Similarly, let $\mathbf{U}=S_{p} H U^{*}, \mathbf{V}=S_{p} \hat{H} V^{*} \in \mathcal{B}^{S^{p} H}$. Then

$$
\begin{aligned}
& \|\mathbf{U}-\mathbf{V}\|_{B}^{2}=\left\|S_{p} \hat{H}\left(U^{*}-V^{*}\right)\right\|_{B}^{2}= \\
& L \max _{l \in[L]}\left\|(U-V) \hat{H}^{*} S_{p} b_{l}\right\|_{2}^{2}=L \max _{l \in[L]}\left\|\sum_{s \in[S]} \hat{h}_{s}^{*} S_{p} b_{l}\left(u_{s}-v_{s}\right)\right\|_{2}^{2} \\
& \leq \operatorname{Lmax}_{l \in[L]} \sum_{s \in[S]}\left|\hat{h}_{s}^{*} S_{p} b_{l}\right|^{2}\left\|u_{s}-v_{s}\right\|_{2}^{2} \leq \mu_{H}^{2} \sum_{s \in[S]}\left\|u_{s}-v_{s}\right\|_{2}^{2} \\
& =\mu_{H}^{2}\|U-V\|_{F}^{2}=\mu_{H}^{2}\left\|\hat{H}\left(U^{*}-V^{*}\right)\right\|_{F}^{2} \\
& =\mu_{H}^{2}\left\|T_{p} S_{p} \hat{H}\left(U^{*}-V^{*}\right)\right\|_{F}^{2},
\end{aligned}
$$

where the last equality holds since $T_{p} S_{p}=I$ and the rest is as in (124). The final norm can be split into

$$
\begin{aligned}
& \mu_{H}^{2}\left\|T_{p} S_{p} \hat{H}\left(U^{*}-V^{*}\right)\right\|_{F}^{2} \leq \mu_{H}^{2}\left\|T_{p}\right\|^{2}\left\|S_{p} \hat{H}\left(U^{*}-V^{*}\right)\right\|_{F}^{2} \\
& \leq \mu_{H}^{2}(1+\nu)^{2}\|\mathbf{U}-\mathbf{V}\|_{F}^{2} \lesssim \mu_{H}^{2}\|\mathbf{U}-\mathbf{V}\|_{F}^{2},
\end{aligned}
$$

where the second inequality is due to $\left\|T_{p}\right\| \leq 1+\nu$. Using Dudley's inequality as in (125), we get

$$
\gamma_{2}\left(\mathcal{B}^{S^{p} H},\|\cdot\|_{B}\right) \lesssim \mu_{H} \sqrt{S N}
$$

To bound $\gamma_{2}\left(\mathcal{B}^{M},\|\cdot\|_{B}\right)$, notice that $d_{B}\left(\mathcal{B}^{M}\right) \leq \sqrt{K} \mu$, so by Dudley's inequality we get

$$
\gamma_{2}\left(\mathcal{B}^{M},\|\cdot\|_{B}\right) \lesssim \int_{0}^{\sqrt{K} \mu} \sqrt{\log N\left(\mathcal{B}^{M},\|\cdot\|_{B}, \epsilon\right)} d \epsilon .
$$

For the rhs, we use Lemma 20 and have

$$
\begin{aligned}
& \gamma_{2}\left(\mathcal{B}^{M},\|\cdot\|_{B}\right) \lesssim \\
& \int_{0}^{\sqrt{K} \mu} \sqrt{\log N\left(B(0,1) \subset \mathbb{R}^{S},\|\cdot\|_{2}, \frac{\epsilon}{2 \sqrt{K} \mu}\right)} d \epsilon+ \\
& \int_{0}^{\sqrt{K} \mu} \sqrt{S \log N\left(B(0,1) \subset \mathbb{C}^{K},\|\cdot\|_{B}, \frac{\epsilon}{2}\right)} d \epsilon
\end{aligned}
$$

Thus, the first integral is bounded by

$$
\begin{aligned}
& \int_{0}^{\sqrt{K} \mu} \sqrt{\log N\left(B(0,1) \subset \mathbb{R}^{S},\|\cdot\|_{2}, \frac{\epsilon}{2 \sqrt{K} \mu}\right)} d \epsilon \leq \\
& \sqrt{S} \int_{0}^{\sqrt{K} \mu} \sqrt{\log \left(1+\frac{4 \sqrt{K} \mu}{\epsilon}\right)} d \epsilon \lesssim \sqrt{S K} \mu,
\end{aligned}
$$

where we have used a standard volumetric estimate and a change of variables. For the second integral in $(130)$, we provide a private case of the derivation in [17] for completeness. We now split the second integral in 130 to two integration intervals: $[0,1]$ and $[1, \sqrt{K} \mu]$. For $\epsilon \in(0,1)$, we define

$$
B(0,1) \subset \sqrt{K} \mu B_{\|\cdot\|_{B}}(0,1) \triangleq\left\{x \in \mathbb{C}^{K} \mid\|x\|_{B} \leq \sqrt{K} \mu\right\} .(132)
$$

This implies that

$$
\begin{aligned}
& N\left(B(0,1) \subset \mathbb{C}^{K},\|\cdot\|_{B}, \epsilon\right) \leq \\
& N\left(B(0,1)_{\|\cdot\|_{B}} \subset \mathbb{C}^{K},\|\cdot\|_{B}, \frac{\epsilon}{\sqrt{K} \mu}\right) \leq\left(1+\frac{2 \sqrt{K} \mu}{\epsilon}\right)^{2 K}
\end{aligned}
$$

where the last inequality is a standard bound for the covering number. For the interval $[0,1]$ we get the following bound

$$
\begin{aligned}
& \int_{0}^{1} \sqrt{S \log N\left(B(0,1) \subset \mathbb{C}^{K},\|\cdot\|_{B}, \frac{\epsilon}{2}\right)} d \epsilon \\
& \leq \sqrt{2 K S} \int_{0}^{1} \sqrt{\log \left(1+\frac{2 \sqrt{K} \mu}{\epsilon}\right)} d \epsilon \\
& \leq \sqrt{2 K S \log (e(1+2 \sqrt{K} \mu))},
\end{aligned}
$$

where the first inequality is due to 133 and the second one is due to Lemma C.9 in [32]. Now we deal with the case where $\epsilon \in(1, \sqrt{K} \mu)$. Using Lemma 21, we get

$$
\begin{aligned}
& \int_{1}^{\sqrt{K} \mu} \sqrt{S N\left(B(0,1) \subset \mathbb{C}^{K},\|\cdot\|_{B}, \frac{\epsilon}{2}\right)} d \epsilon \lesssim \\
& \int_{1}^{\sqrt{K} \mu} \frac{\sqrt{S K \log (L)} \mu}{\epsilon} d \epsilon \lesssim \sqrt{S K \log (L)} \mu \log \left(K \mu^{2}\right) .
\end{aligned}
$$

Combining (134) with (135) provides us with

$$
\begin{aligned}
& \int_{0}^{\sqrt{K} \mu} \sqrt{S \log N\left(B(0,1) \subset \mathbb{C}^{K},\|\cdot\|_{B}, \frac{\epsilon}{2}\right)} d \epsilon \lesssim \\
& \sqrt{S K \log (L)} \mu \log \left(K \mu^{2}\right),
\end{aligned}
$$

where we use the fact that 135 is the dominant interval. Plugging (131) and (136) in (130), and considering again the dominant part, leads to

$$
\gamma_{2}\left(\mathcal{B}^{M},\|\cdot\|_{B}\right) \lesssim \sqrt{S K \log (L)} \mu \log \left(K \mu^{2}\right)
$$

The result stated in (58) is given by the summation of the three bounds in (125), 128) and 137). 


\section{Proof of Lemma 20}

Proof of Lemma 20, For all $s \in[S]$, let $\mathcal{N}_{s}$ be an $\frac{\epsilon}{2}$-cover of $B(0,1) \subset \mathbb{C}^{K}$ with respect to the $\|\cdot\|_{B}$-norm and $\mathcal{O}$ be an $\frac{\epsilon}{2 \sqrt{K \mu}}$-cover of $B(0,1) \subset \mathbb{R}^{S}$ with respect to the $\|\cdot\|_{2}$ norm. We will show now that any $X=U \hat{M}^{*} \in \mathcal{B}^{M}$ can be approximated by $Y=\sum_{s \in[S]} \sigma_{s} v_{s} \hat{m}_{s}^{*}$ where $\sigma \in \mathcal{O}$ and $v_{s} \in \mathcal{N}_{s}$. Notice that the number of such $Y \mathrm{~s}$ is bounded by the right hand side of the inequality in 119 . Thus, it remains to show that such a construction is possible. Since $\sigma \in \mathcal{O}$, we may pick it to satisfy

$$
\sqrt{\sum_{s \in[S]}\left(\left\|u_{s}\right\|_{2}-\sigma_{s}\right)^{2}} \leq \frac{\epsilon}{2 \sqrt{K} \mu} .
$$

Notice, that $\left(\left\|u_{1}\right\|_{2}, \ldots,\left\|u_{S}\right\|_{2}\right) \in B(0,1)$, since $X \in \mathcal{B}^{M}$ and $\hat{M}$ is orthonormal. In a similar way, since $v_{s} \in \mathcal{N}_{s}$, we select it such that

$$
\left\|\frac{1}{\left\|u_{s}\right\|_{2}} u_{s}-v_{s}\right\|_{B} \leq \frac{\epsilon}{2}
$$

for all $s \in S$. Thus, for $\hat{Y}=\sum_{s \in[S]}\left\|u_{s}\right\|_{2} v_{s} \hat{m}^{*}$, we have

$$
\begin{aligned}
& \|X-\hat{Y}\|_{B}^{2}=\left\|\sum_{s \in[S]}\left(u_{s}-\left\|u_{s}\right\|_{2} v_{s}\right) \hat{m}_{s}^{*}\right\|_{B}^{2} \\
& =\operatorname{Liax}_{l \in[L]}\left\|\sum_{s \in[S]} \hat{m}_{s}\left(u_{s}-\left\|u_{s}\right\|_{2} v_{s}\right)^{*} b_{l}\right\|_{2}^{2} \\
& =\operatorname{Liax}_{l \in[L]} \sum_{s, k \in[S]} b_{l}^{*}\left(u_{s}-\left\|u_{s}\right\|_{2} v_{s}\right) \hat{m}_{s}^{*} \hat{m}_{k}\left(u_{k}-\left\|u_{k}\right\|_{2} v_{k}\right)^{*} b_{l} \\
& =\operatorname{Lmax}_{l \in[L]} \sum_{s \in[S]} b_{l}^{*}\left(u_{s}-\left\|u_{s}\right\|_{2} v_{s}\right)\left(u_{s}-\left\|u_{s}\right\|_{2} v_{s}\right)^{*} b_{l}
\end{aligned}
$$

where the second equality is due to the definition of $\|\cdot\|_{B}$ in (50) combined with the non-negativity of the norm, and the last step follows the orthonormality of $\hat{M}$. Next, we continue to bound

$$
\begin{aligned}
& \operatorname{Lmax}_{l \in[L]} \sum_{s \in[S]} b_{l}^{*}\left(u_{s}-\left\|u_{s}\right\|_{2} v_{s}\right)\left(u_{s}-\left\|u_{s}\right\|_{2} v_{s}\right)^{*} b_{l} \\
& \leq \sum_{s \in[S]} \operatorname{Lmax}_{l \in[L]}\left(b_{l}^{*}\left(u_{s}-\left\|u_{s}\right\|_{2} v_{s}\right)\left(u_{s}-\left\|u_{s}\right\|_{2} v_{s}\right)^{*} b_{l}\right) \\
& =\sum_{s \in[S]}\left\|u_{s}-\right\| u_{s}\left\|_{2} v_{s}\right\|_{B}^{2}=\sum_{s \in[S]}\|\| u_{s}\left\|_{2}\left(\frac{1}{\left\|u_{s}\right\|_{2}} u_{s}-v_{s}\right)\right\|_{B}^{2} \\
& \leq \frac{\epsilon^{2}}{4} \sum_{s \in[S]}\left\|u_{s}\right\|_{2}^{2}=\frac{\epsilon^{2}}{4}\|U\|_{F}^{2} \leq \frac{\epsilon^{2}}{4}
\end{aligned}
$$

where the first equality is again due to due to the definition of $\|\cdot\|_{B}$ in 50 combined with the non-negativity of the norm, the second inequality is due to (139) and the last step holds since $X=U \hat{M}^{*} \in \mathcal{B}^{M}$ is normalized and $\hat{M}$ is orthonormal. To conclude this step, by 140, 1141) we have

$$
\|X-\hat{Y}\|_{B}^{2} \leq \frac{\epsilon^{2}}{4}
$$

To complete the proof, we similarly have

$$
\begin{aligned}
& \|\hat{Y}-Y\|_{B}^{2}=\left\|\sum_{s \in[S]}\left(\left\|u_{s}\right\|_{2}-\sigma_{s}\right) v_{s} \hat{m}_{s}^{*}\right\|_{B}^{2} \leq \\
& \sum_{s \in[S]}\left\|\left(\left\|u_{s}\right\|_{2}-\sigma_{s}\right) v_{s}\right\|_{B}^{2}=\sum_{s \in[S]}\left(\left\|u_{s}\right\|_{2}-\sigma_{s}\right)^{2}\left\|v_{s}\right\|_{B}^{2} \leq \\
& \sum_{s \in[S]} K \mu^{2}\left(\left\|u_{s}\right\|_{2}-\sigma_{s}\right)^{2} \leq \frac{\epsilon^{2}}{4}
\end{aligned}
$$

where again we used the orthonormlity of $\hat{M}$, the nonnegativity of the norm and the fact that

$$
\begin{aligned}
\left\|v_{s}\right\|_{B} & =\sqrt{L} \max _{l \in L}\left|v_{s}^{*} b_{l}\right| \\
& \leq \sqrt{L}\left\|v_{s}\right\|_{2} \max _{l \in L}\left\|b_{l}\right\|_{2} \leq \sqrt{K} \mu,
\end{aligned}
$$

which holds since $v_{s} \in \mathcal{N}_{s}$. Finally, by combining (142) and 143, we get $\|X-Y\|_{B} \leq \epsilon$.

\section{ACKNOWLEDGMENT}

We thank the anonymous reviewers for their useful comments that helped to improve the paper. This research was supported by ERC-StG grant no. 757497 (SPADE).

\section{REFERENCES}

[1] J. Liu, J. Xin, Y. Qi, and F.-G. Zheng, "A time domain algorithm for blind separation of convolutive sound mixtures and 11 constrainted minimization of cross correlations," Communications in Mathematical Sciences - COMMUN MATH SCI, vol. 7, 012009.

[2] N. Shamir, Z. Zalevsky, L. Yaroslavsky, and B. Javidi, "Blind source separation of images based on general cross correlation of linear operators," Journal of Electronic Imaging - J ELECTRON IMAGING, vol. 20, 042011.

[3] S. Shwartz, Y. Y. Schechner, and M. Zibulevsky, "Efficient separation of convolutive image mixtures," in Independent Component Analysis and Blind Signal Separation, J. Rosca, D. Erdogmus, J. C. Príncipe, and S. Haykin, Eds. Berlin, Heidelberg: Springer Berlin Heidelberg, 2006, pp. 246-253.

[4] Xiaodong Wang and H. V. Poor, "Blind equalization and multiuser detection in dispersive cdma channels," IEEE Transactions on Communications, vol. 46, no. 1, pp. 91-103, Jan 1998.

[5] G. Wunder, H. Boche, T. Strohmer, and P. Jung, "Sparse signal processing concepts for efficient $5 \mathrm{~g}$ system design," Access, IEEE, vol. 3, 11 2014.

[6] S. Ling and T. Strohmer, "Blind deconvolution meets blind demixing: Algorithms and performance bounds," IEEE Transactions on Information Theory, vol. 63, no. 7, pp. 4497-4520, July 2017.

[7] S. Haykin, Blind Deconvolution, ser. Prentice-Hall information and system sciences series. PTR Prentice Hall, 1994. [Online]. Available: https://books.google.sh/books?id=KO1SAAAAMAAJ

[8] S. Choudhary and U. Mitra, "Fundamental limits of blind deconvolution part I: ambiguity kernel," CoRR, vol. abs/1411.3810, 2014. [Online]. Available: http://arxiv.org/abs/1411.3810

[9] P. Walk, P. Jung, G. Pfander, and B. Hassibi, "Ambiguities on convolutions with applications to phase retrieval,' in 2016 50th Asilomar Conference on Signals, Systems and Computers, 2016, pp. 1228-1234.

[10] K. Lee, Y. Wu, and Y. Bresler, "Near-optimal compressed sensing of a class of sparse low-rank matrices via sparse power factorization," IEEE Transactions on Information Theory, vol. 64, no. 3, pp. 1666-1698, 2018.

[11] K. Lee, Y. Li, M. Junge, and Y. Bresler, "Blind recovery of sparse signals from subsampled convolution," IEEE Transactions on Information Theory, vol. 63, no. 2, pp. 802-821, 2017.

[12] S. Oymak, A. Jalali, M. Fazel, Y. C. Eldar, and B. Hassibi, "Simultaneously structured models with application to sparse and low-rank matrices," IEEE Transactions on Information Theory, vol. 61, no. 5, pp. 2886-2908, 2015. 
[13] A. Levin, Y. Weiss, F. Durand, and W. T. Freeman, "Understanding and evaluating blind deconvolution algorithms," in 2009 IEEE Conference on Computer Vision and Pattern Recognition, 2009, pp. 1964-1971.

[14] A. Ahmed, B. Recht, and J. K. Romberg, "Blind deconvolution using convex programming," IEEE Transactions on Information Theory, vol. 60, pp. 1711-1732, 2014.

[15] E. J. Candès, T. Strohmer, and V. Voroninski, "Phaselift: Exact and stable signal recovery from magnitude measurements via convex programming," Communications on Pure and Applied Mathematics, vol. 66, no. 8, pp. 1241-1274, 2013.

[16] E. J. Candès and B. Recht, "Exact matrix completion via convex optimization," Foundations of Computational Mathematics, vol. 9, no. 6, p. 717, Apr 2009. [Online]. Available: https://doi.org/10.1007/ s10208-009-9045-5

[17] P. Jung, F. Krahmer, and D. Stöger, "Blind demixing and deconvolution at near-optimal rate," IEEE Transactions on Information Theory, vol. 64, no. 2, pp. 704-727, Feb 2018.

[18] X. Li, S. Ling, T. Strohmer, and K. Wei, "Rapid, robust, and reliable blind deconvolution via nonconvex optimization," Applied and Computational Harmonic Analysis, vol. 47, no. 3, pp. 893 - 934, 2019. [Online]. Available: http://www.sciencedirect.com/science/article/ pii/S1063520318300149

[19] S. Ling and T. Strohmer, "Regularized gradient descent: a non-convex recipe for fast joint blind deconvolution and demixing," Information and Inference: A Journal of the IMA, vol. 8, no. 1, pp. 1-49, 032018. [Online]. Available: https://doi.org/10.1093/imaiai/iax022

[20] J. Dong and Y. Shi, "Nonconvex demixing from bilinear measurements," IEEE Transactions on Signal Processing, vol. 66, no. 19, pp. 5152-5166, 2018.

[21] T. Strohmer and K. Wei, "Painless breakups-efficient demixing of low rank matrices," Journal of Fourier Analysis and Applications, vol. 25, no. 1, pp. 1-31, 2019.

[22] J. Dong, K. Yang, and Y. Shi, "Blind demixing for low-latency communication," IEEE Transactions on Wireless Communications, vol. 18 , no. 2, pp. 897-911, 2019.

[23] J. Dong and Y. Shi, "Blind demixing via wirtinger flow with random initialization," in Proceedings of Machine Learning Research, ser. Proceedings of Machine Learning Research, K. Chaudhuri and M. Sugiyama, Eds., vol. 89. PMLR, 16-18 Apr 2019, pp. 362-370.

[24] J. Dong, Y. Shi, and Z. Ding, "Blind over-the-air computation and data fusion via provable wirtinger flow," IEEE Transactions on Signal Processing, vol. 68, pp. 1136-1151, 2020.

[25] Y. Chi, Y. M. Lu, and Y. Chen, "Nonconvex optimization meets lowrank matrix factorization: An overview," IEEE Transactions on Signal Processing, vol. 67, no. 20, pp. 5239-5269, 2019.

[26] A. Ahmed, "A convex approach to blind mimo communications," IEEE Wireless Communications Letters, vol. 7, no. 5, pp. 812-815, Oct 2018.

[27] K. Lee, Y. Li, M. Junge, and Y. Bresler, "Stability in blind deconvolution of sparse signals and reconstruction by alternating minimization," in 2015 International Conference on Sampling Theory and Applications (SampTA), 2015, pp. 158-162.

[28] D. Gross, "Recovering low-rank matrices from few coefficients in any basis," IEEE Transactions on Information Theory, vol. 57, no. 3, pp. 1548-1566, 2011.

[29] M. Talagrand, Upper and Lower Bounds for Stochastic Processes: Modern Methods and Classical Problems, ser. Ergebnisse der Mathematik und ihrer Grenzgebiete. 3. Folge / A Series of Modern Surveys in Mathematics. Springer Berlin Heidelberg, 2016. [Online] Available: https://books.google.co.il/books?id=JjQGvgAACAAJ

[30] S. Burer and R. Monteiro, "A nonlinear programming algorithm for solving semidefinite programs via low-rank factorization," Mathematical Programming, Series B, vol. 95, pp. 329-357, 022003.

[31] R. Dudley, "The sizes of compact subsets of hilbert space and continuity of gaussian processes," Journal of Functional Analysis, vol. 1, no. 3, pp. 290 - 330, 1967. [Online]. Available: http: //www.sciencedirect.com/science/article/pii/0022123667900171

[32] S. Foucart and H. Rauhut, A Mathematical Introduction to Compressive Sensing. Springer, 2013 